\title{
KU LEEUVEN
}

\section{Machine Consciousness and the Global Workspace Theory}

MohammadHossein Manuel Haqiqatkhah

\author{
Thesis submitted for the degree of \\ Master of Science in Artificial \\ Intelligence, option Engineering and \\ Computer Science \\ Thesis supervisor: \\ Prof. Roger Vergauwen \\ Assessors: \\ Prof. Bettina Berendt \\ Prof. Danny De Schreye
}




\section{(c) Copyright KU Leuven}

Without written permission of the thesis supervisor and the author it is forbidden to reproduce or adapt in any form or by any means any part of this publication. Requests for obtaining the right to reproduce or utilize parts of this publication should be addressed to the Departement Computerwetenschappen, Celestijnenlaan 200A bus 2402, B-3001 Heverlee, +32-16-327700 or by email info@cs .kuleuven. be.

A written permission of the thesis supervisor is also required to use the methods, products, schematics and programmes described in this work for industrial or commercial use, and for submitting this publication in scientific contests. 


\section{Preface}

This thesis is humbly dedicated to the following persons who might not know how immeasurably I owe them-and a green leaf is the whole endowment of the dervish.

Mīrzā Mahdī Gharawī Iṣfahānìi,

whose nudge inspired me to delve into philosophy of mind;

Garrett Kiriakos-Fugate,

who had a key role in settling my inner clashes;

Mohammad KhoshZabān,

without whom I would have never found what I had lost; and

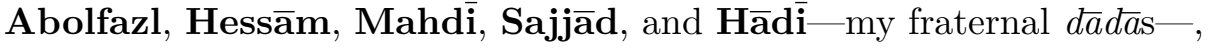

who were always there when I needed them.

I would like to sincerely thank my lovely parents - without whose support I could not have reached this far - and my heartening brother - whom I consider my role model.

And thanks to Agnes Moors, Ilias Rentzeperis, and AmirHossein Sālehī for their valuable comments.

MohammadHossein Manuel Haqiqatkhah 


\section{Contents}

Preface $\quad$ i

Abstract iii

List of Figures and Tables iv

1 Introduction $\quad 1$

1.1 Notions of Consciousness . . . . . . . . . . . . . . . . . . 2

1.2 Problems of Consciousness . . . . . . . . . . . . . . . . . 4

1.3 Machine Consciousness . . . . . . . . . . . . . . 5

2 Explanation, Explication, and Levels 9

2.1 The Answers That Satisfy and Those Which Fail to . . . . . . . . 10

2.2 TLMs and Mechanistic Explanations . . . . . . . . . . . . . 13

2.3 Some Philosophical Notes on the ETLM . . . . . . . . . . . . . 16

2.4 Other Levels . . . . . . . . . . . . . . . . . . . . 19

3 Neurocognitive Theories of Consciousness 2

3.1 From Neural Correlates to Models of Consciousness . . . . . . . . . . 26

3.2 The Global Workspace Theory of Consciousness . . . . . . . . . . . 28

3.3 Global Workspace Models: The Realizations of the GWT . . . . . . 34

3.4 A Closer Look at the GWT . . . . . . . . . . . . . . . . 38

4 Computational Implementations of the Global Workspace Theory 43

4.1 IDA . . . . . . . . . . . . . . . . . . . 44

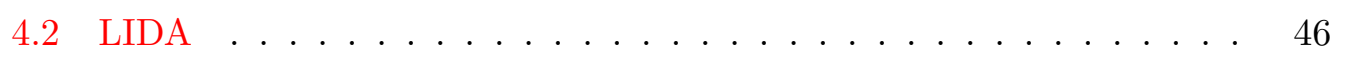

5 Can Machines Ever Become Conscious? 49

5.1 The Frame Problem: A Touchstone for (Models of) Consciousness . 50

5.2 The Frame Problem in a GW Architecture . . . . . . . . . . . . 53

6 Conclusion $\quad 57$

$\begin{array}{ll}\text { References } & 59\end{array}$ 


\section{Abstract}

The past century has seen a resurgence of interest in the study of consciousness among scholars of various fields, from philosophy to psychology and neuroscience. Since the birth of Artificial Intelligence in the 1950s, the study of consciousness in machines has received an increasing amount of attention in computer science that gave rise to the new field of machine consciousness (MC).

Meanwhile, interdisciplinary research in philosophy, neuroscience, and cognitive science has advanced neurocognitive theories for consciousness. Among many models proposed for consciousness, the Global Workspace Theory (GWT) is a promising theory of consciousness that has received a staggering amount of philosophical and empirical support in the past decades.

This dissertation discusses the GWT and its potentials for MC from a mechanistic point of view. To do so, Chapter 1 gives an overview of the philosophical study of consciousness and the history of MC. Then, in Chapter 2, mechanistic explanations and tri-level models are introduced, which provide a robust framework to construct and assay various theories of consciousness.

In Chapter 3, neural correlates (and thereby, neurocognitive theories) of consciousness are introduced. This chapter presents the GWT in details and, along with its strengths, discusses the philosophical issues it raises. Chapter 4 addresses two computational implementations of the GWT (viz., IDA and LIDA) which satisfy specific goals of MC. Finally, in Chapter 5, one of the philosophical problems of MC, namely, the Frame Problem (FP), is introduced. It is argued that the architectures based on the GWT are immune to the FP. The chapter concludes that the GWT is capable of "solving" the FP, and discusses its implications for MC and the computational theory of mind. Chapter 6 wraps up the dissertation by reviewing the content. 


\section{List of Figures and Tables}

\section{List of Figures}

2.1 Phenomenon and its mechanism . . . . . . . . . . . . . . . 12

2.2 Hierarchical mechanistic explanation . . . . . . . . . . . . . . . 12

2.3 Extended tri-level model . . . . . . . . . . . . . . . . . 15

2.4 Causation in mechanistic explanation . . . . . . . . . . . . . . . . 20

2.5 Levels of organization in computer engineering and biology . . . . . . . 21

2.6 Levels of organization in nervous system . . . . . . . . . . . . . . . 22

2.7 Levels of abstraction in cognitive neuroscience . . . . . . . . . . . 23

3.1 Global workspace as a bottleneck and broadcaster . . . . . . . . . . . . 29

3.2 Two aspects of global workspace as a stage . . . . . . . . . . . . . . . 29

3.3 Schematic diagram of global access . . . . . . . . . . . . . . . . . 32

3.4 Small-world structures in graph theory . . . . . . . . . . . . . . . 37

3.5 Global neural workspace in brain . . . . . . . . . . . . . . 39

4.1 The IDA architecture . . . . . . . . . . . . . . . . 45

4.2 The LIDA cognitive cycle . . . . . . . . . . . . . . . . . 47

5.1 A naïve model of serialized information flow . . . . . . . . . . . . 54

5.2 The global workspace model of information flow . . . . . . . . . . 55

\section{List of Tables}

2.1 Latin terms for explanation and explication . . . . . . . . . . . 11 


\section{Chapter 1}

\section{Introduction}

The anecdotes of minstrel and wine utter and little seek the mystery of eon; For, this enigma, one did-and shall-not determine by sagacity.

- Ḥִ̂̄FEZ OF SHĪRĀZ (CA. 1315-1390)

There are not many scientific and technological advancements that have mesmerized the lay people as much as artificial intelligence does. The idea of humanoid machines that can manifest human features has haunted minds of people for centuries. The birth of modern computer science in the 20th century annunciated a future wherein machines can do any mundane job as long as it is algorithmically defined. The emergence of the new field of artificial intelligence (AI) in the 1950s, accompanied by the exponential progress in computing machinery in the coming decades, set hopes in development of machine that could manifest (or possess) humane characteristics: to understand, think, and feel-the features attributed to consciousness by philosophers. Now computer science and philosophy of mind found themselves in an arena to cross swords - and forge alliances.

Consciousness is a highly ambiguous concept which, to many, is one of the most mysterious phenomena in the world; above all, it is one of the last redoubts not fully conquered by scientific explanation - and some argue that it is, in essence, "well beyond the realm of human understanding." Consciousness, as an aspect of the human mind, constitutes our inner, most private mental lives with the most private subjective experiences of ourselves and the world. Theories of consciousness, whether philosophical or scientific, have always been subject to heated debates. The philosophical disputes around matters are not always about the explanans ${ }^{1}$ (e.g. "what

\footnotetext{
The epigraphs of Ḥâfez in all chapters are adapted from (Wilberforce Clarke, 1891).

${ }^{1}$ The terms explanan and explicans (and their plural forms, explanans and explicantia, respectively) will be used frequently in this manuscript. They refer to the explanation or explication given for a particular phenomenon. It is crucial to note that explanation and explication have different meanings; the former focuses on giving (causal) explanations about the underlying nature of the phenomenon, while the latter is a form of detailed, systematic description of it (Audi, 2015). The differences between these two will be discussed in Chapter 2, Section 2.1.
} 
consciousness is"); in many cases, they stem from the conceptual inexactness in the definitions of the explananda ${ }^{2}$.

This thesis investigates how the global workspace theory can be applied to machine consciousness. The current introductory chapter starts with a philosophical taxonomy to reduce conceptual sloppiness around the terms "conscious" and "consciousness." Then Van Gulick's (2018) classification of the problems that any account of consciousness needs to address are succinctly reviewed. These sections aim neither at discussing the problems in details nor reviewing the theories; the only reason to enumerate them is to help us in connecting the implications of the global workspace theory to specific aspects of consciousness. Later in the chapter, the field of machine consciousness (MC) is introduced, and the main strands of MC research are mentioned. The chapter concludes by sketching the structure of the rest of the dissertation.

Before moving on, it is crucial to note that the reader shall not expect an exhaustive treatment of the issues discussed in this thesis - that is beyond the purview of such manuscript. Moreover, since the obscurity of the desiderata ${ }^{3}$ demands being placed within a broader perspective, addressing the peripheral matters is inevitable. The reader might find, for instance, the (rather) detailed treatment of the neural correlates of consciousness irrelevant to the overall aim of the thesis. Nonetheless, such contexts are required to elucidate the global workspace theory and its contribution to MC.

\subsection{Notions of Consciousness}

One can use the adjective "conscious" in two different ways: transitively or intransitively. Its transitive use refers to either conscious access to, or conscious processing of, a specific piece of information" (as in "Mark was not conscious of his own pain"). This is referred to as content consciousness $\left(C_{\text {content }}\right.$; Dehaene \& Changeux, 2011). The intransitive use of the adjective can either refer to whether a creature is conscious (creature consciousness $\left[C_{\text {creature }}\right]$ ), or whether a mental state is consciously experienced (state consciousness $\left[C_{\text {state }}\right]$ ). The word "consciousness" is usually the noun form of the adjective conscious. As such, consciousness, as a noun, "is not typically treated as a substantive entity but merely the abstract reification of whatever property or aspect is attributed by the relevant use of the adjective 'conscious'" (Van Gulick, 2018, sec. 2.3, para 2). Some, on the other hand, treat consciousness as a definite entity with an ontological status which is not in the focus

\footnotetext{
${ }^{2}$ The terms explanandum and explicandum (and their plural forms, explananda and explicanda, respectively) refer to the phenomena that are to be explained or explicated. See footnote 1.

${ }^{3}$ Plural for desideratum (derived from Latin word dēsīderātum), meaning "something for which a desire or longing is felt; something wanting and required or desired." (Oxford English Dictionary, n.d.)

${ }^{4}$ Here it is implied that the contents of (consciousness) mental states are of an informational nature - which is a controversial stance. This will be addressed in Chapter 2. See (Pitt, 2018) for a discussion.
} 
of this dissertation. With this introduction, the different meanings of the adjective conscious are explained in the following.

\subsubsection{Creature consciousness}

This notion, an intransitive adjective denoted by $C_{\text {creature }}^{p}$, applies to the ability of an object (or, more specifically, an organism) to have property $p$ or a manifestation of $p$. This notion can be used in two distinct ways. First, the creature is $C_{\text {creature }}^{p}$ if it is capable of having or exhibiting property $p$ (as in "Trees can feel pain hence they are conscious.") Additionally, the creature is $C_{\text {creature }}^{p}$ if it is exhibiting property $p$ at a specific time and place (as in "Mary's father was still conscious when she rushed into the room.") Note that the latter use is about the state of the creature as a whole at a particular moment in time, and it should not be confused with $C_{\text {state }}$ (which is about mental states, not an overall state of the creature.)

The most important of these properties, based upon Van Gulick (2018, sec. 2.1), listed from the least to the most demanding, include the following: sentience (being capable of sensing and responding to its surrounding); wakefulness (having or showing a distinct state of vigilance); self-consciousness or self-awareness 5 (being "not only aware but also aware that [it is] aware, thus treating creature consciousness as a form of self-consciousness"; Van Gulick, 2018, sec. 2.1, para. 4); and what-it-is-like-ness (existence of "'something that it is like' to be that creature, i.e., some subjective way the world seems or appears from the creature's mental or experiential point of view as Nagel (1974) introduced for the first time.”; Van Gulick, 2018, sec. 2.1, para. 5).

Conceptualization of $C_{\text {creature }}$, in any of its different senses, is not facile. For instance, the criteria of specific properties (e.g., sentience or wakefulness) are not well-defined; "boundaries may be blurry, and intermediate cases may be involved. For example, is one conscious in the relevant sense when dreaming, hypnotized or in a fugue state?" (Van Gulick, 2018, sec. 2.1, para. 3) Some other properties, such as self-consciousness and what-it-is-like-ness are, in essence, hard to define since they are surrounded by yet more complicated philosophical issues.

\subsubsection{State consciousness}

This notion, when used as an intransitive adjective, applies to properties of mental states rather than the (potential or actual) properties of the creature. A mental state can be said to be $C_{\text {state }}^{p}$ if it has property $p$. Van Gulick (2018, sec. 2.2) mentions six types of mental states that can be considered state conscious: states one is aware of (those that one is aware of having, "[involving] a form of meta-mentality or meta-intentionality in so far as they require mental states that are themselves about mental states."; sec. 2.2, para. 2); qualitative states (those having or involving "qualitative or experiential properties of the sort often referred to as 'qualia' or "raw sensory feels" "; sec. 2.2, para. 3); phenomenal states ("overall structure of experience and involves far more than sensory qualia, encompassing much of the

\footnotetext{
${ }^{5}$ One should be wary that self-consciousness and self-awareness can take different connotations given certain interpretations. See (Van Gulick, 2018, sec. 2.1, para. 4) for a short discussion.
} 
spatial, temporal and conceptual organization of our experience of the world and of ourselves as agents in it"; sec. 2.2, para. 4); what-it-is-like state (if there is something like to be in that state); access consciousness (if the state is available "to interact with other states of its kind and of the access that one has to its content"; sec. 2.2, par. 6) and narrative consciousness ("appealing to the notion of the "stream of consciousness', regarded as an ongoing more or less serial narrative of episodes from the perspective of an actual or merely virtual self"; sec. 2.2, par. 7).

Again, like $C_{\text {creature }}$, defining $C_{\text {state }}$ is not straightforward. For instance, the definition of $C_{\text {state }}^{\text {qualtive }}$ heavily relies on one's stance toward qualia, the nature - and even existence - of which is subject to much disagreement (Tye, 2018; Van Gulick, 2018, sec. 2.2, par. 3).

\subsubsection{Content consciousness}

As discussed, creatures and mental states can be conscious in multiple senses. Though, in some cases, we are more concerned about the content of a mental state rather than the mental state itself. Dehaene and Changeux (2011) define content consciousness $\left(C_{\text {content }}\right)$ as a transitive adjective for processes of information that are subject to the current inner experience of the creature and are reportable "verbally or by an intended gesture." (p. 200) As will be discussed in Chapter 3, while many other processes co-occur at the same time, only a limited amount of information can be consciously accessed, delineating the current conscious content of the mind.

There is a fine line between $C_{\text {state }}^{\text {access }}$ and $C_{\text {content }}$ which should not be missed. $C_{\text {state }}^{\text {access }}$ is a property of mental states, whereas $C_{\text {content }}$ is a feature of mental processes. The memory of one's first kiss can be $C_{\text {state }}^{\text {access }}$ if, for instance, its content can be accessed by another memory of her first date. The process of remembering the memory of the night will be $C_{\text {content }}$ if it is actively in the scope of her attention.

\subsection{Problems of Consciousness}

As we have seen, the term "consciousness" stands for various aspects of mind where each can be approached from various angles, hence understanding it requires addressing multiple explanans and explicantia. Even if one isolates different meanings of consciousness (e.g., $C_{\text {creature }}^{\text {sentience }}$ ) into individual explananda and explicanda, single explanans and explicantia will not grant a thorough understanding of the matter; the angles from which the phenomena can be approached limit the kind of answers that can be derived.

For the case of $C_{\text {creature }}^{\text {sentience }}$, for instance, different questions can be asked, each requiring their own explanation (or explications): What does it mean to be a (non)sentient creature (explicans)? How does a creature become sentient (explanan)? Why a creature need to be sentient, and what function does sentience serve for the creature (explicans)? Van Gulick (2018) calls these questions (that concern description, explanation, and function, respectively), the what-, how-, and whyquestions. These questions are discussed briefly in what follows, and more details can be found in (Van Gulick, 2018, secs. 3-6), upon which this section is written. 
The descriptive (what) questions concern the principal features of consciousness in any of its senses and seek answers that can delineate creatures, states, or contents that have certain forms of consciousness from those who lack them. This delineation of features of consciousness is not possible without solid descriptions of more fundamental phenomena. For instance, one has to probe the difference between first-person, subjective perception and the third-person, "external" assessment of mental states before defining $C_{\text {state }}^{\text {awareness }}$. Other what-questions are about describing the qualitative character of the conscious states (and how they form the phenomenal structure of our experience), the unity of our conscious experiences, and aboutness ${ }^{6}$ of the mental states.

The explanatory questions, on the other hand, ask "how consciousness of the relevant sort comes to exist. Is it a primitive aspect of reality, and if not, how does (or could) consciousness in the relevant respect arise from or be caused by nonconscious entities or processes?" (Van Gulick, 2018, sec. 3, para. 3). The questions in this category are among the most controversial topics in philosophy of mind. Most importantly, before providing any explanation for different sorts of consciousness, one has to take a stance towards the explanatory gap: Is there any way, potentially or actually, to give objective, empirical explanations for mental states and entities which are essentially subjective? The questions about this gap-most importantly, whether it exists, to what extent it poses limits to our understanding of consciousness, and what the answers to these questions would imply-have polarized theories of consciousness - although there is no consensus at either side of the spectrum. Another polarizing matter in answering the how-questions is the degree to which the descriptions have to be reductive. For a more detailed discussion, see (Van Gulick, 2018, sec. 5).

Finally, the questions of functions (why) probe the significance of consciousness for creatures; does it serve any function for the creature, and if so, does that entail any causal role for the consciousness of that sort in the universe? The why questions can be solely about functions of consciousness (e.g., its role in providing a factual representation of the state of affairs for the creature, or easing its social coordination), or they can address more fundamental questions on the nature of free will, selfhood, and causal status of mental processes and states. See (Van Gulick, 2018, sec. 6).

The problems mentioned so far have been asked quite differently in more pragmatic (and less philosophical) literature of neurophilosophy, which will be discussed in Chapter 3.

\subsection{Machine Consciousness}

The study of consciousness has been a diverse field wherein questions outnumber answers. Yet, another line of research, namely machine consciousness (MC), has emerged to ask questions about the relation between algorithmic processes - in which

\footnotetext{
${ }^{6}$ Aboutness is a less technical term for intentionality, which is often defined as "the power of minds and mental states to be about, to represent, or to stand for, things, properties and states of affairs." (Jacob, 2019, para. 1) It will not be discussed in this manuscript any further.
} 
machines outperform humans - and the features attributed to consciousness - what had been believed to be unique to humans. Interestingly, the audaciousness of the early MC research has benefitted the philosophical and scientific understanding of human consciousness.

Since the second half of the 20th century, there has been a resurgence of interest in human and animal consciousness across different disciplines; from philosophy to psychology and neuroscience. Parallel to this, a new field in computer science was emerging to enable machines to "intelligently" do tasks not explicitly programmed into them. Perhaps the most impactful spark of the idea that machines can possess intelligence was Alan Turing's paper in Mind (1950) in which he suggested that an "imitation game" can be a benchmark of "thinking" in machines, wherein the behavior of a machine is assessed by an interrogator. Since thinking was believed to be a conscious process, his proposal gained fame in consciousness research as the Turing Test and has been subject to heated debate since (for a detailed review of the matter, see Oppy \& Dowe, 2019). Though, the official beginning of the field under the title "artificial intelligence" is attributed to the 1956 Dartmouth Conference where the term was first coined and later popularized. ("Artificial intelligence (AI) coined at dartmouth," n.d.) For a detailed historical account of AI (and Turing's role in it), see (Bringsjord \& Govindarajulu, 2018; González, 2007, Chapters 1-2; Haikonen, 2003, Chapter 2).

In his review of the history of MC, David Gamez (2008) explains how the line between (areas of) AI and consciousness research has become blurry in the past decades:

People have also started to test theories of consciousness using computer models and there has been some speculation that this could eventually lead to more intelligent machines that might actually have phenomenal states. This type of research is gradually becoming known as "machine consciousness," although "artificial consciousness" and occasionally "digital sentience"1 have also been used to describe it. Each of these terms has their own merits, but the growing number of meetings dedicated to "machine consciousness" suggests that this is likely to become the standard name for the field. (p. 887)

Due to the heterogeneity of the field, Gamez (2008) stratifies MC into four lines of research: implementations that exhibit external behaviors associated with consciousness (MC1); emulation of cognitive processes associated with consciousness (MC2); implementation of architectures suggested to be causes or correlates of consciousness (MC3); and phenomenal consciousness in machine (MC4).

This classification is beneficial in the study of MC as a discipline; it allows including a wide range of research under the umbrella term of machine consciousness without taking them equally accountable for ambitious claims made by the field as a whole. Moreover, it helps understand and evaluate the criticism raised against MC and the common misconceptions among the critics.

The remainder of this dissertation is organized as follows. Chapter 2 addresses explanation and explication, and introduces a tri-level mechanistic explanations of 
intelligent beings. These models help demarcate various levels of explanations in the study of consciousness. In Chapter 3 the neuroscientific and neurocognitive theories of consciousness are introduced. The global workspace theory (GWT), the core subject of the thesis, is discussed in this chapter. Chapter 4 takes a look at the computational models of the GWT and shows that they meet certain criteria of MC. Chapter 5 addresses an important criticism against machine consciousness, namely, the Frame Problem, and argues that the GWT can solve that problem. Finally, Chapter 6 summarizes the thesis. 



\section{Chapter 2}

\section{Explanation, Explication, and Levels}

What is this lofty, smooth-yet, much-embossed-roof [of sky]? In the world, acquainted with this mystery, sage there is none.

- ḤĀFeZ of ShĪRĀZ (CA. 1315-1390)

David Marr, in his seminal book Vision (1982), suggested a tri-level hypothesis for different levels of analysis of intelligent organisms. His proposal, along with his theory of stages of vision, greatly influenced cognitive psychology and neuroscience. Although leukemia did not let him live past the age of 35, he set cornerstones in cognitive science and computational modeling of perception and cognition. His work also inspired AI, especially the field of computer vision. Marr's tri-level hypothesis (MTLH), the central tenet of his cognitive theory, sheds light on different aspects of "intelligence" ${ }^{1}$ and perception by laying down a framework for mechanistic explanations of cognition. Later interpretations (or extensions) of his hypothesis covered broader scopes of explanation. This is the incentive to make use of the family of tri-level models (TLMs) in answering the problems of consciousness - and machine consciousness.

To properly understand TLMs, their relevance, and their significance, one must first make a substantial distinction between explanations and explications and see what would quench her curiosity about the desideratum - and what would not. Section 2.1 tries to somewhat illuminate the matter and introduce a class of explanations that empower TLMs. Section 2.2 is the mainstay of the chapter and discusses levels of analysis and TLMs. First, MTLH and its interpretations are introduced. Then an extended variant of Marr's original model is presented in details. Later, in Section 2.3, multiple realizability and two chief critics against representationalism (which is at the heart of TLMs) are addressed and some important philosophical notions (viz., emergence, supervenience, and causality) are briefly touched upon. Finally, in Section 2.4, the concepts of levels of abstraction and levels of organization are

\footnotetext{
${ }^{1}$ See footnote 8 further in this chapter.
} 
contrasted with the levels of analysis as they are needed for the discussion in Chapter 3, Section 3.3.

It should be noted that this chapter is a modest effort in utilizing the insights of TLMs to peek in some of the most profound issues in the philosophy of biology and neuroscience. Excellent systematic discussions on the issues surrounding (mechanistic) explanations in (neuro)science can be found in works of Carl Craver and colleagues (e.g., Craver, 2001, 2007; Craver \& Tabery, 2019; Machamer, Darden, \& Craver, 2000).

\subsection{The Answers That Satisfy and Those Which Fail to}

The scientific inquiry (or analysis) mostly starts with a phenomenon $P$ that seeks answers to questions about the nature of $P$, and its relation to other phenomena in the world, which can be parts of $P$ or distinct entities. This is also the case for many of the problems philosophers try to address. Such answers are the longed-for desiderata of the inquiry - what have been wished for. Sometimes - if not most of the time - the desiderata are vaguely outlined. Quite often, this non-specificity is partly the reason why people disagree on whether some seemingly reasonable answers are sufficient for the questions at hand. Chapter 1 tried to make the problems of consciousness more specifically defined by stratifying them into classes of what-, how-, and why-questions. This section introduces two types of analysis and then discusses mechanistic explanations in more depth.

\subsubsection{Explanations vs. explications}

The desiderata, according to Audi (2015), can be classified into two categories: those concerning what it is for $P$ to be or occur in a certain way (i.e., explication); and those accounting for why (or how) $P$ happens to be what it is (i.e., explanation):

[There are] two main ways for a philosophical theory to account for some phenomenon. It can give an explanation of it or an explication of it. To explain something is to say why it exists or has the properties it does. But some things are brute; they admit of no explanation. Nevertheless, brute phenomena may be susceptible of explication. It is sometimes possible to say in an illuminating way what it is for something to exist or to have certain properties, even though there is nothing to be said about why. (p. 208)

In practice, explanation usually leans towards addressing some sort of causal account for the underpinnings of $P$ and tries to uncover what is going on under the hood. Conversely, explication can be thought of as a more detailed descriptive response to characterizing $P$. The word analysis can be used as an umbrella term for explanation and explication. The Latin terms used to refer to such questions and answers were introduced Chapter 1, and they are reviewed in Table 2.1 to avoid confusion. 
2.1. The Answers That Satisfy and Those Which Fail to

TABLE 2.1: Latin terms for explanation and explication

\begin{tabular}{llllll}
\hline & \multicolumn{2}{c}{ Explanation } & & \multicolumn{2}{c}{ Explication } \\
\cline { 2 - 3 } \cline { 5 - 6 } & Singular & Plural & & Singular & Plural \\
\hline Question/Phenomenon & explanandum & explananda & explicandum & explicanda \\
Answer/Desideratum & explanan & explanans & & explicans & explicantia \\
\hline
\end{tabular}

\subsubsection{Mechanistic explanations}

Mechanistic explanation (ME) of a phenomenon $P$ (which is often manifested as a "behavior" of system $S$ ) explains $P$ by virtue of causal relations between parts of $S$. A mechanism underlying phenomenon $P$ "consists of entities and activities organized in such a way that they are responsible for the phenomenon." ${ }^{2}$ (Illari \& Williamson, 2012, p. 120)

Craver's (2007) canonical visual representation of such mechanism (fig. 4.6, shown in Figure 2.1 below) is illuminating. As Craver and Tabery explain:

At the top is the phenomenon, some system $S$ engaged in behavior $\Psi$. This is the behavior of the mechanism as a whole. Beneath it are the parts (the $X \mathrm{~s}$ ) and their activities (the $\phi \mathrm{s}$ ) organized together. The dotted roughly-vertical lines reflect the fact that the parts and activities are contained within, are components of, the mechanism engaged in this behavior. Thus represented, mechanisms are decompositional in the sense that the behavior of the system as a whole can be broken down into organized interactions among the activities of the parts. (2019, sec. 2)

An ME is a viable explanan for an explanandum only if it addresses its four elements adequately ${ }^{3}$ : the phenomenon (the black box $[\mathrm{BB}]$ ), the parts, causation, and the organization ${ }^{4}$. The criterion of "adequately addressing the elements" has two significant implications:

$\mathbf{I}_{\mathbf{1}}$. An ME need not make realist commitments to the ontological status of its elements - e.g., an explanan can be valid even if its $X$ s are imaginary, non-"existing" abstractions; and

$\mathbf{I}_{2}$. Whether an explanan is valid or not is ultimately a subjective decision.

$\mathrm{I}_{1}$ and $\mathrm{I}_{2}$ have yet another remarkable implication:

$\mathbf{I}_{\mathbf{3}}$. ME can be realized in different ways.

\footnotetext{
${ }^{2}$ This can be considered as an "ecumenical stance" toward the definition of mechanism. See (Craver \& Tabery, 2019, sec. 2) for a detailed comparison of conceptualizations of mechanism.

${ }^{3}$ The adverb adequately is crucial here.

${ }^{4}$ See Section 2.4 .
} 


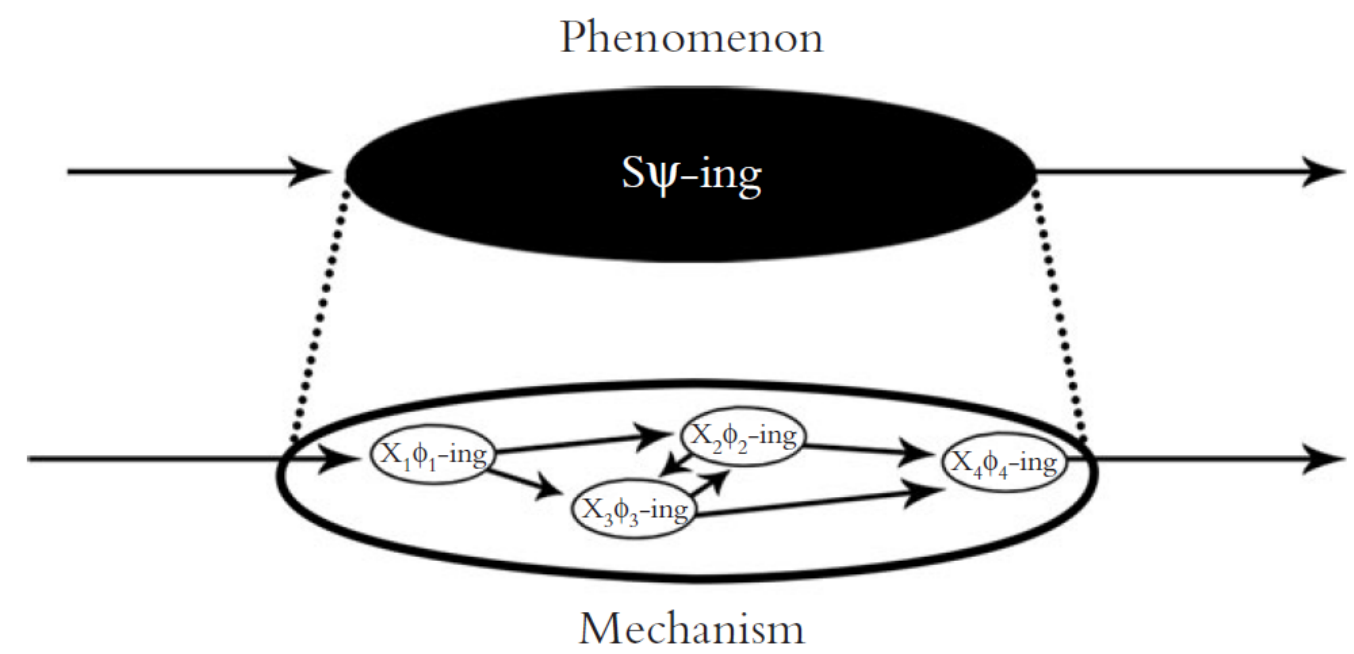

FIGURE 2.1: Mechanistic explanation of a phenomenon Top: A phenomenon $P$ exhibiting $\Psi$. Bottom: The underlying mechanism of $P$ : its parts $(X \mathrm{~s})$ with their activities ( $\phi$ s). Adapted from (Craver, 2007, fig. 4.6).

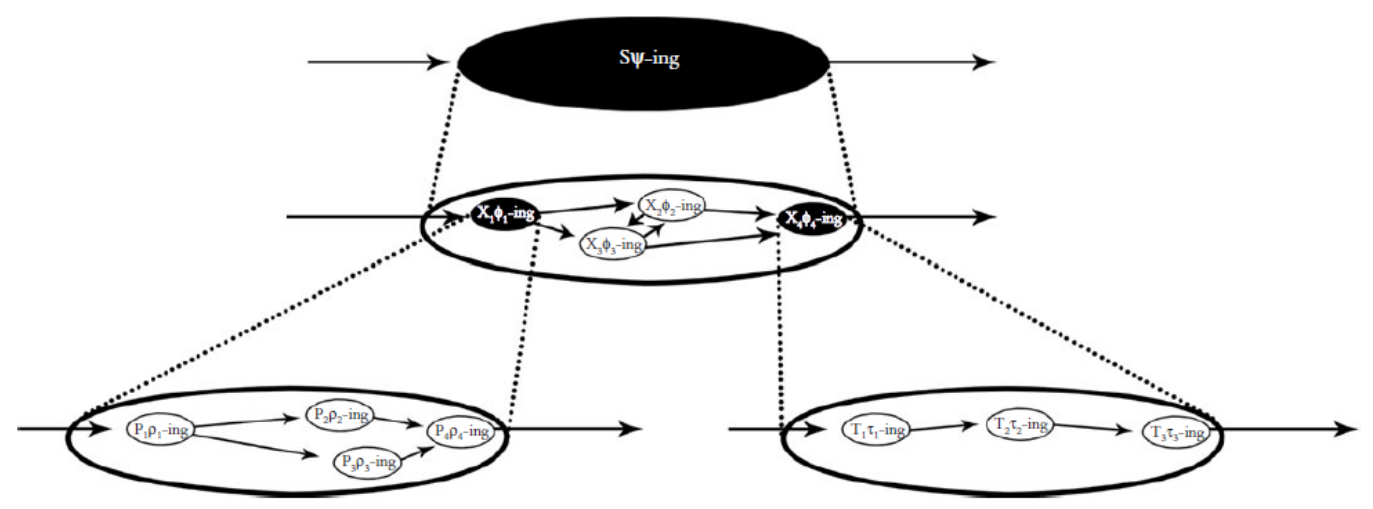

FIGURE 2.2: Hierarchical mechanistic explanation A phenomenon $P$ can be mechanistically explained by its parts $X \mathrm{~s}$. The $X \mathrm{~s}$ can be further explained as lower-level phenomena. Adapted from (Craver, 2007, fig. 5.8).

$\mathrm{I}_{3}$ is famously known as the multiple realizability (MR) thesis in philosophy of mind (Bickle, 2019).

Given $\mathrm{I}_{2}$, one may make a more fine-grained $\mathrm{ME}$ by treating the parts (i.e., $X \mathrm{~s}$ ) as new explananda (i.e., BBs), each having their own MEs. This leads to a hierarchical ME, as shown in Figure 2.2 (adapted from Craver, 2007, fig. 5.8).

Given MR, alternative MEs (including those with more in-depth mechanistic explanations) can be compared based on the trade-off between their scope and their 
parsimony ${ }^{5}$. Section 2.2 makes use of MEs at different levels of analysis.

\subsection{TLMs and Mechanistic Explanations}

Marr laid down a mechanistic account in the analysis of information-processing systems (IPSs). In his opinion, such systems, including conscious creatures, can be described (and further analyzed) from three distinct perspectives. Peebles and Cooper (2015) write:

"Marr's 'trilevel hypothesis' ... that information-processing systems can be analyzed in terms of the problems that they solve (Marr's computational level), the representations and processes by which they solve them (the algorithmic and representational level), and the physical instantiation of these representations and processes (the implementation level) has been reformulated several times in the subsequent 30 years ... and remains a core tenet of cognitive science." (pp. 187-188)

A classic example of MTLH is a pocket calculator. Suppose one presses buttons $3,+$, 7 , and $=$, and the calculator displays 10 on its screen. This IPS can be analyzed in three levels as follows. At the computational level, the IPS has performed an addition of those numbers. At the second level, the IPS has made symbolic representations of the numbers and did an addition operation on those symbols. At the implementation level, the IPS has used logic gates in its hardware to perform such operation. One can make the aforementioned explanations more specific (or more fine-grained) by analyzing how the operation at each level has been carried out. For instance, one can investigate how such symbols were represented at the second level, or look more closely how the logic gates were implemented in the hardware. We will get back to this later in the chapter.

There are other formulations of these levels. For instance, "Zenon Pylyshyn (1984) calls them the semantic, the syntactic, and the physical; and textbooks in cognitive psychology sometimes call them the levels of content, form, and medium (e.g., Glass et al. 1979)." (McClamrock, 1991, p. 185). In what follows, another formulation of TLMs is introduced.

\subsubsection{Extending Marr's model}

As an alternative interpretation (or formulation) of MTLH, the extended tri-level model (ETLM) uses the terms functional, mental, and realization for the three levels. The functional level ( $\left.L_{\text {functional }}\right)^{6}$ is equivalent to the highest level of analysis, known as the level of computation, semantics, or content in previous formulations. The

\footnotetext{
${ }^{5}$ Parsimonious (i.e., "simple") models and theories are favored by a methodological principle known as Ockham's Razor. See, e.g., (Baker, 2016).

${ }^{6}$ Some might suggest that the term observation level suits the highest level better. However, "observability" is not limited to the highest level as one can make measurements (and observations) at the $L_{\text {realization. }}$
} 
mental level ( $\left.L_{\text {mental }}\right)$, the intermediate level of algorithm, syntax, and forms, is where mental representations and constructs are related via operations. Finally, the realization level $\left(L_{\text {realization }}\right)^{7}$, the closest level to the (neural) substrate, can be equated to the medium mentioned above or physical level. A general case of ETLM is shown graphically in Figure 2.3 and described below.

At the $L_{\text {functional }}$, the "intelligent" being $(\mathrm{IB})^{8}$ is treated as a $\mathrm{BB}$ that exhibits a particular "behavior" or output ( $f_{o}$ for the output at the $\left.L_{\text {functional }}\right)$ as a result of ${ }^{9}$ (or in response to) a particular input ( $f_{i}$ for the input at the $\left.L_{\text {functional }}\right) . f_{i}$ can be of purely sensory kind (e.g., tactile sensation, visual stimuli) or of more complicated forms which may or may not be reduced to purely sensory stimuli. Examples of the latter are complex, compound, or multifaceted sensory stimuli, or stimuli of higher orders of intricacy, such as behaviors (either by the IB itself at an earlier time or by other beings). The input can also be non-existent, i.e., the IB exhibits a particular behavior without any specific, distinguishable input. This can be the case if, for instance, $f_{i}$ had occurred before the observation took place, or if $f_{i}$ lies within the IB itself.

The IB can be analyzed solely at the $L_{\text {functional, }}$, and such analysis would be sufficient if one believes that "behave is what organisms do" (see Graham, 2019). However, one can give a mechanistic explanation for the BB. This ME belongs to the second level of analysis, namely, $L_{\text {mental }}$.

At the $L_{\text {mental }}$, the phenomenon $P$ - exhibited by the BB of $L_{\text {functional }}$ - can be explained as a mechanism consisting of mental objects: mental representations $\left(M_{\text {representation }} \mathrm{s}\right)$ and mental processes $\left(M_{\text {process }} \mathrm{s}\right)$ connecting them. $M_{\text {representation }} \mathrm{s}$ can be thought of as "[patterns] of information or encoding that denotes something." (Reggia, 2013, p. 122) In the cognitive sciences, $M_{\text {representation }}$ s are conventionally assumed to be symbolic objects, and $M_{\text {process }}$ s to be algorithmic ${ }^{10}$ (Pitt, 2018). The information content, hence $M_{\text {representation }}$ s, do not need to be symbolic; one can conceptualize information qualitatively, either semantically (e.g., Poggiolesi, 2015) or as states of the entity (e.g., van Benthem \& van Rooy, 2003). See (Adriaans, 2019) for a detailed discussion and further references.

One can give further MEs for the $M_{\text {representation }}$ s by treating them as BBs at $L_{\text {mental }}$. Getting back to the example of the pocket calculator, the symbols of 3 and 7 are represented by 0011 and 0111 , respectively. The addition operation itself is done symbolically using bitwise AND and XOR operators to get to the binary representation of 10, i.e., 1010. We call this implementation IMP1.

\footnotetext{
${ }^{7}$ The term implementation is strictly avoided here since it can also refer to the algorithmic implementation of a symbolic operation in $L_{\text {mental }}$, without any ties to the $L_{\text {realization }}$.

${ }^{8}$ For better generalizability, other terms, such as "organism," "human person," "information processing system," or "conscious being" have been avoided. Note that the adjective intelligent is used loosely here and shall not imply any relationship between different definitions of intelligence and different aspects of creature or state consciousness discussed in Chapter 1.

${ }^{9}$ The phrase "as a result of" should be used with caution to avoid causal implications - such association between $f_{i}$ and $f_{o}$ is not necessarily a causal relationship.

${ }^{10}$ The word algorithm has a dominant connotation of being a computational set of processes. Hence, algorithm is often used interchangeably with computational.
} 


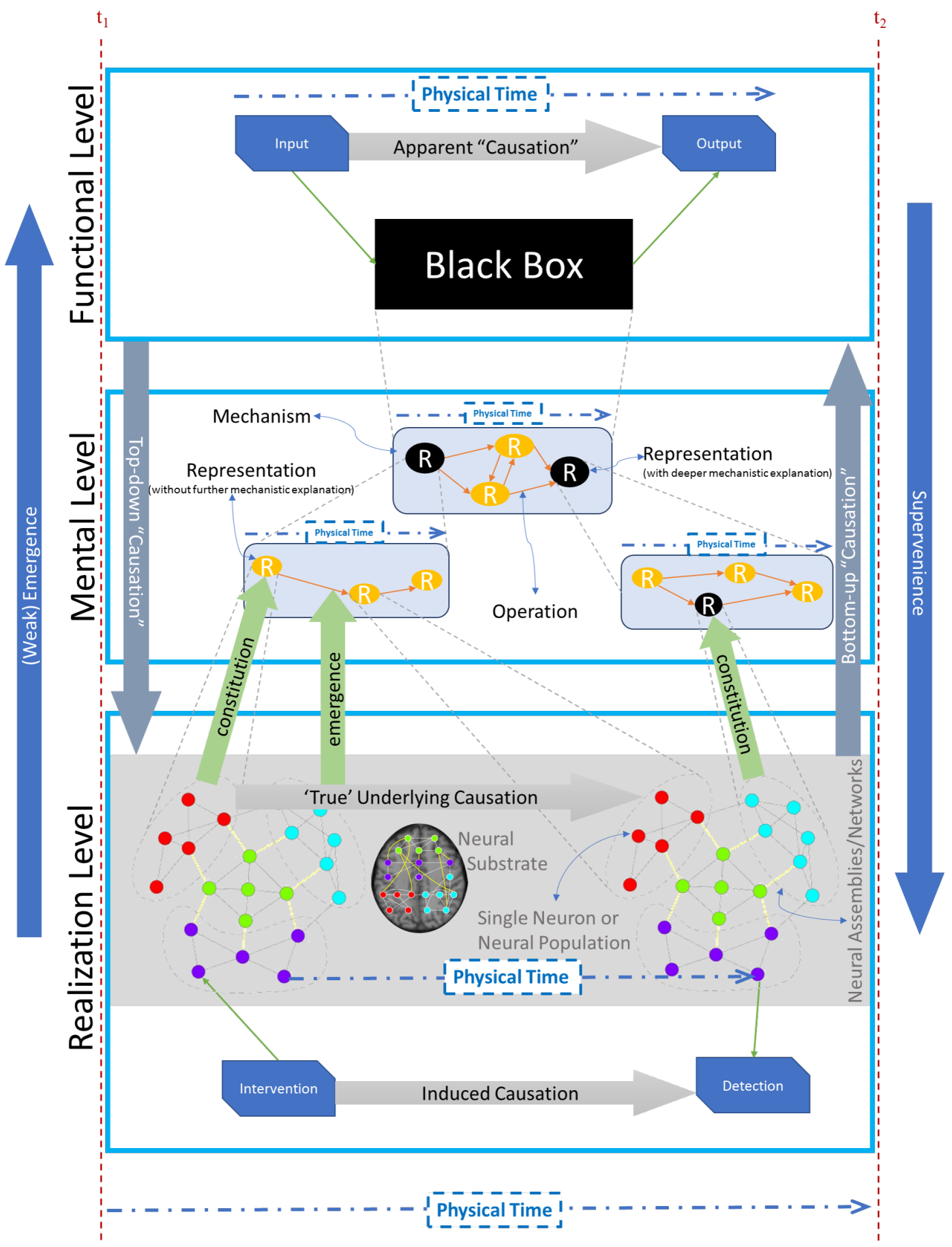

FIGURE 2.3: Extended tri-level model for an organism See Sections 2.2 .1 and 2.3 for explanation. The diagram of the brain network (in the realization level) is adapted from (Giessing \& Thiel, 2012, fig. 1). 
At the $L_{\text {realization }}$ mechanisms underlying representations and operations of the $L_{\text {mental }}$ are realized in a physical medium. For the case of the calculators, the representations are realized by electrical signals with different voltage levels, and the operators are realized using logic gates. One can give deeper explanation and tell how such gates themselves are realized, i.e., with transistors in an electronic circuit.

\subsection{Some Philosophical Notes on the ETLM}

There are several issues that demand a closer look: multiple realizability, some criticism against the representational account, intra-level (vertical) relations and causation in ETLM, and the limitations of ETLM. These issues are discussed below.

\subsubsection{Multiple realizability and the ETLM}

Given the discussion in section 2.1.2, the same phenomenon $P$ can be (mechanistically) explained in multiple ways (cf. $\mathrm{I}_{3}$ ). For instance, for the case of the pocket calculator, another symbolic mechanism can also carry out the addition operation; the same operation can be equivalently implemented using bitwise NOT and AND operators (IMP2), or by the different placement of the same AND and XOR operators (IMP3). Moreover, the numbers could have been represented by other symbolic representations, such as in hexadecimal numeric system: $3_{16}+7_{16}=A_{16}$. This alternative representation requires an alternative operation. Furthermore, the hexadecimal representation (and operation) could be further represented (and operated) with the binary alternatives, e.g., with that of IMP1 (as discussed in Section 2.2.1). These are trivial cases of MR, where one implementation can be easily reduced to another. This is not necessarily the case as the alternative representational explanations can be formulated in totally different ways.

The $L_{\text {realization }}$ can also be an arena for MR. For instance, the same IMP1 could be realized using different electronic circuits; there are multiple ways to design a circuit to perform AND and XOR operations with the same transistors. Moreover, other circuitry could have been used; with different electronic devices, with vacuum tubes, or even mechanically - although the latter realizations would no longer fit in ordinary pockets.

Due to MR, there is no unique representational implementation or physical realization (hence ME) for the same function. This implies that the true mechanism is often not identifiable. The only ways to know which candidate ME is the actual mechanism of $P$ are either through a priori knowledge of how the system has been "designed," or through a retrospective look at the physical realization of the system with background knowledge of what representations such realization can produce. Without such knowledge, we can only, pragmatically, compare the candidate proposals (according to some criterion, such as those mentioned in Section 2.1.2) to find the most probable ME among them-yet there is no guarantee whether the true ME lies among the set of proposals. ${ }^{11}$

\footnotetext{
${ }^{11}$ In statistical modeling, the goal is to estimate the processes underlying the measurements. In
} 
It is more complicated in consciousness studies for at least three reasons: (1) The "design" process of the brain -if we can assume it is indeed a product of any specific design - is not (yet) clear; (2) Our knowledge of the neuroscience of the brain is not mature enough; and (3) we do not have such a priori knowledge - we do not even know whether such representations actually exist. It is likely that in some (distant) future we can overcome the first two issues, but the third problem is unlikely to be settled anytime soon - if ever.

\subsubsection{Two critiques of the representational account}

The representational account of cognitive science has been attacked by two strands of critics: those who deny the existence of such representations, and those who skepticize its premises - most importantly, the symbolic and informational nature of such representations. Pitt notes an example of the latter:

Van Gelder (1995) denies that psychological processes are computational. He argues that cognitive systems are dynamic, and that cognitive states are not relations to mental symbols, but quantifiable states of a complex system consisting of (in the case of human beings) a nervous system, a body and the environment in which they are embedded. Cognitive processes are not rule-governed sequences of discrete symbolic states, but continuous, evolving total states of dynamic systems determined by continuous, simultaneous and mutually determining states of the systems' components. Representation in a dynamic system is essentially information-theoretic, though the bearers of information are not symbols, but state variables or parameters. (Pitt, 2018, sec. 8)

Such objections, in and of themselves, might not invalidate the representationalist account present in ETLM. Because the mechanisms are multiply realizable $\left(\mathrm{I}_{3}\right)$, multiple mechanisms can be candidate explanans for $P$. Moreover, given $\mathrm{I}_{1}$, the $M_{\text {representation } \mathrm{S} \text { can be purely imaginary }}{ }^{12}$, and similarly, the $M_{\text {process }}$ s can be noncomputational $^{13}$; the informational content of such representations can be nonsymbolic (cf. 2.2.1, para. 4). Thus, the nature of such representations does not necessarily have to be symbolic.

Neither those two objections against the representationalist account are the only ones, nor the responses provided thereof are (sufficiently) robust against further critiques. For a detailed review of the dispute around representationalism, see (Pitt, 2018).

doing so, different applications (e.g., statistical inference or prediction) and different assumptions (e.g., whether the actual model is among the candidate models) determines whether (and how) we can identify the model or make a decision about the most reasonable candidate. For further reading, see (Claeskens \& Hjort, 2008, Chapter 4).

${ }^{12}$ Hence the earlier use of the term "object" to refer to them does not posit any ontological status for them.

${ }^{13} \mathrm{cf}$. footnote 11. 


\title{
2.3.3 Vertical relations in the ETLM
}

The explanatory power of a multi-level, mechanistic model depends on explaining how elements of one level relate to those of others. Such relations can be called vertical relations. The most important of such relations are supervenience, emergence, and constitution. All of these notions are notorious philosophical concepts that require detailed investigations in relation to ontological dependence (e.g., cf. McLaughlin \& Bennett, 2018; O'Connor \& Wong, 2015; Tahko \& Lowe, 2016; Varzi, 2019). Without delving into details, the first two are explained succinctly here.

Supervenience is a form of directed dependence, as McLaughlin and Bennett (2018) define it,

\begin{abstract}
A set of properties $A$ supervenes upon another set $B$ just in case no two things can differ with respect to $A$-properties without also differing with respect to their $B$-properties. In slogan form, "there cannot be an $A$-difference without a $B$-difference" (p. 1)
\end{abstract}

A classic example of supervenience is the phase (e.g., solid, liquid, gas) of a substance (e.g., water) and its molecular arrangement. The liquidity of water supervenes upon its molecular arrangement. Liquidity strictly depends on such arrangement but is not caused by it; there is no temporal order between such molecular arrangement and the phase of water - any change in one is together with a change in the other. Nonetheless, it is a directed dependence; the phase of water would not change unless the arrangement of its molecules changes, not vice versa.

Emergence happens when an entity exhibits specific properties that are not present in its (putative) parts. Emergence can have two forms, weak and strong. Strong emergence posits that the properties of the whole cannot be reduced to those of its parts. On the other hand, weak emergence states that the emergent properties of the whole are no more than those of its parts. Weak emergence is closely tied to supervenience (Beckermann, 1992).

In the ETLM, the higher levels supervene on the lower levels. As discussed earlier in this chapter, the $M_{\text {representation }}$ s can be recursively cut into smaller pieces to give more fine-grained mechanistic explanations. At some point, an $M_{\text {representation }}$ can be reduced to certain physical properties, where the representation is merely an abstraction of the neural properties (e.g., certain neural activities in certain brain regions). This is the case where the neural properties constitute the $M_{\text {representation }}$. Finally, if the mechanistic explanation is detailed enough, it can be said that the processes in $L_{\text {mental }}$ have weakly ${ }^{14}$ emerged from some processes in the physical substrate of the brain.

\footnotetext{
${ }^{14}$ Given the properties of the mechanistic account in explanation, it is hard to imagine the phenomenon $P$ being anything more than its parts, the relations between them, and their organization. This implies that there remains no room for strong emergence in the ME.
} 


\subsubsection{Causation in the ETLM}

The last (but not least) important relation in ETLM is causation. In this framework, causation takes place within levels horizontally; the "cause" and its effect are distinct "entities," both belonging to the same level, therein the former precedes the latter and the latter depends on the former. This is called horizontal causation. In the $L_{\text {functional }}$, the IB reacts to an input; in the $L_{\text {mental }}$, one $M_{\text {representation }}$ leads to another; and in the $L_{\text {realization }}$, a neural stimulation causes a change in a process or state somewhere in the brain.

In the ETLM, the causation at higher levels eventually (and inevitably) translates, through emergence and supervenience, to the causes at the $L_{\text {realization }}$-and contrariwise. Hence, the "true" causation can only be found at the $L_{\text {realization }}$; this is the very foundation upon which the mechanistic explanations have been built.

The inter-level, vertical "causation," either upwards or downwards, is hence a combination of horizontal causations, supervenience, and emergence. Four possible cases of such relation are shown in Figure 2.4. See (Craver, 2007, sec. 4.8) for a more detailed treatment of the matter in the form of experiments.

\subsubsection{Limitations of the ETLM}

There are three sets of limitation to this account towards IBs as it was presented in this chapter. First and foremost, ETLM posits that the IB is indeed mechanistically explainable, hence rules out several controversial issues such as strong emergenceone can be accused of petitio principii (i.e., begging the question) if she tries to use ETLM to explain, e.g., qualia ${ }^{15}$. Secondly, the model is not precisely defined, and the notions (such as constitution) are sloppy. Finally, the model is silent in many cases. It does not address many questions: How do mental states relate to mental representations? Is mental state $S_{1}$ identical to an $M_{\text {representation }}$, or a combination of $M_{\text {representations }}$ and $M_{\text {process }}$ ? How would $S_{1}$ differ from another mental state $S_{2}$ ?

\subsection{Other Levels}

Before concluding this chapter, attention should be drawn to the difference between the closely interrelated - yet distinct - usages of the levels metaphor. So far in this chapter, levels of analysis have been discussed. Here, two other notions, as used in this dissertation, are explained briefly: levels of organization, and levels of abstraction. ${ }^{16}$

In TLMs, the metaphor of levels of analysis is an epistemic stratification of explanation and description to make room for mechanistic explanations. Levels of organization, on the other hand, as Oppenheim and Putnam (1958) coined it, is an ontic stratification of the phenomenon according to the mereology of its parts into a hierarchy of organization (see Baxendale, 2016; Kersten, 2016, Chapter 2).

\footnotetext{
${ }^{15}$ For instance, Block (1992) criticizes Dennett and Kinsbourne (1992) for a similar reason.

${ }^{16}$ These definitions lack specificity and preciseness. For more careful discussions, see (Craver, 2007, Chapter 5; Kersten, 2016, Chapter 2).
} 
2. Explanation, Explication, and Levels

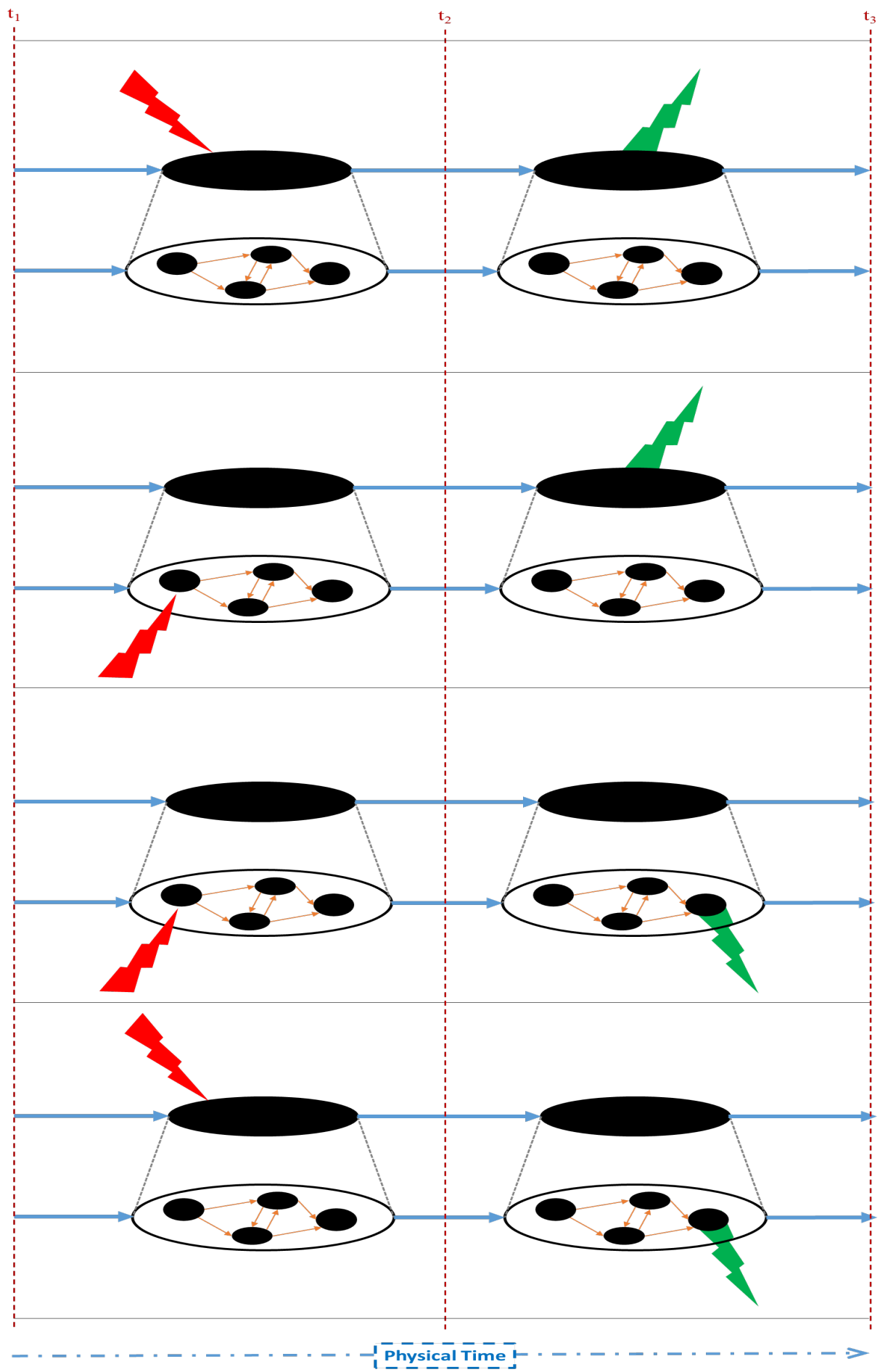

FIGURE 2.4: Causation across levels of mechanistic explanation The intervention in phenomenon $P$ or its parts $X \mathrm{~s}$ is shown with red arrows, and the effect is "observed"/manifested in $P$ or $X \mathrm{~s}$ is shown with green arrows. Note that in ETLM, both $P$ and $X$ s can belong to $L_{\text {mental }}$. See Section 2.3.4 for more explanation.

20 

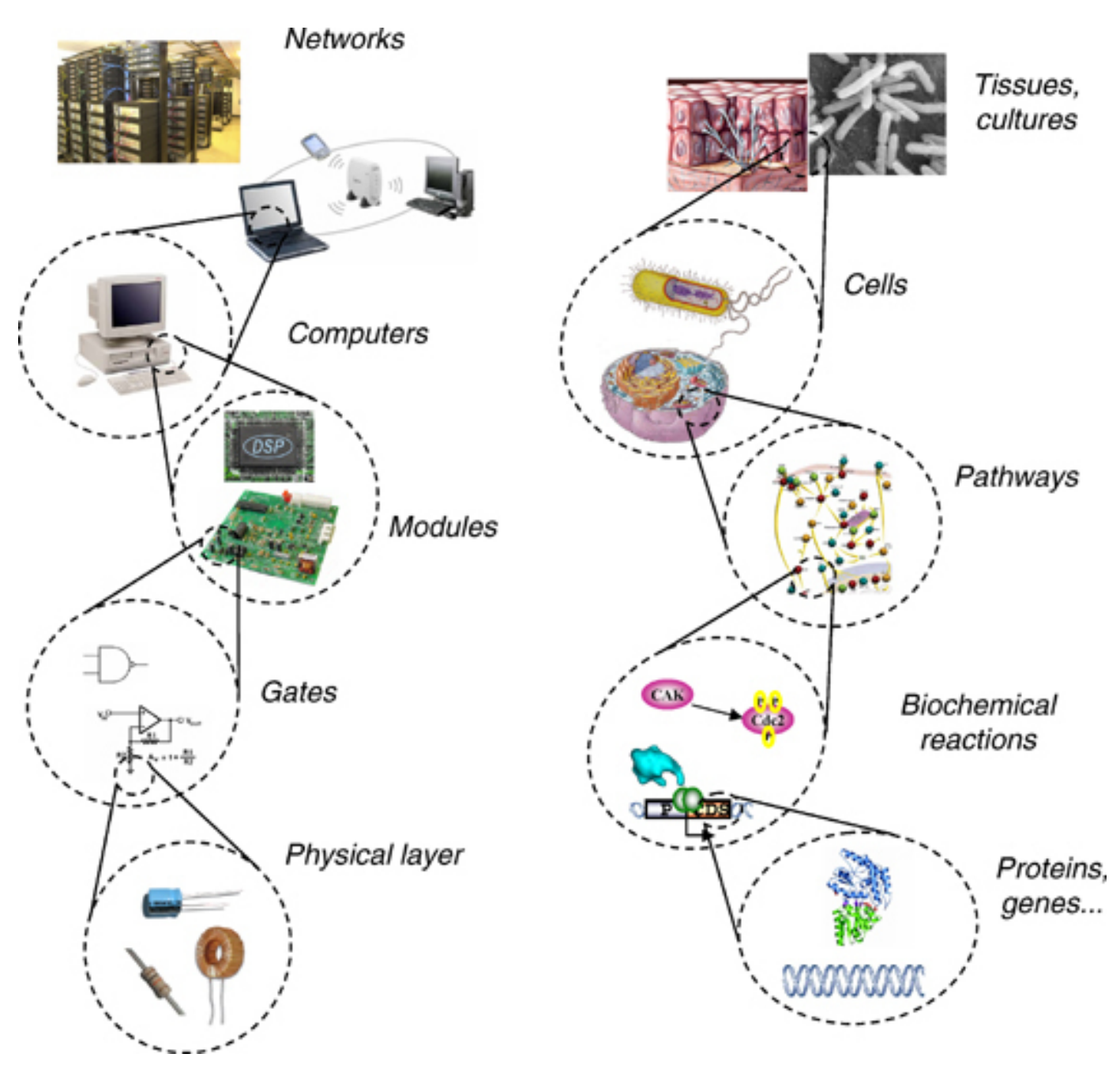

FIGURE 2.5: Levels of organization in computer engineering and biology

Left: A computer network can be studied at different levels: network of computers as a graph of connected devices; devices with certain characteristics and behaviors; hardware elements of computers; logic circuits of the hardware (e.g., the processors); and the electronic elements of the circuits (e.g., transistors). Right: A living tissue can be analyzed at multiple levels: organization of cells within the tissue; internal structure of cells; the biological processes within the cell; biochemical reactions of those processes; structure of genes and proteins involved in such reactions. Adapted from (Andrianantoandro et al., 2006, fig. 1).

Levels of organization can be detected in a variety of fields. Figure 2.5 (adapted from Andrianantoandro, Basu, Karig, \& Weiss, 2006, fig. 1) depicts examples of such levels in computer networks and biology. Figure 2.6 (adapted from Craver, 2007, fig. 5.5) shows these levels in the human nervous system.

Levels of abstraction, on the other hand, are theoretical, semantic stratification 

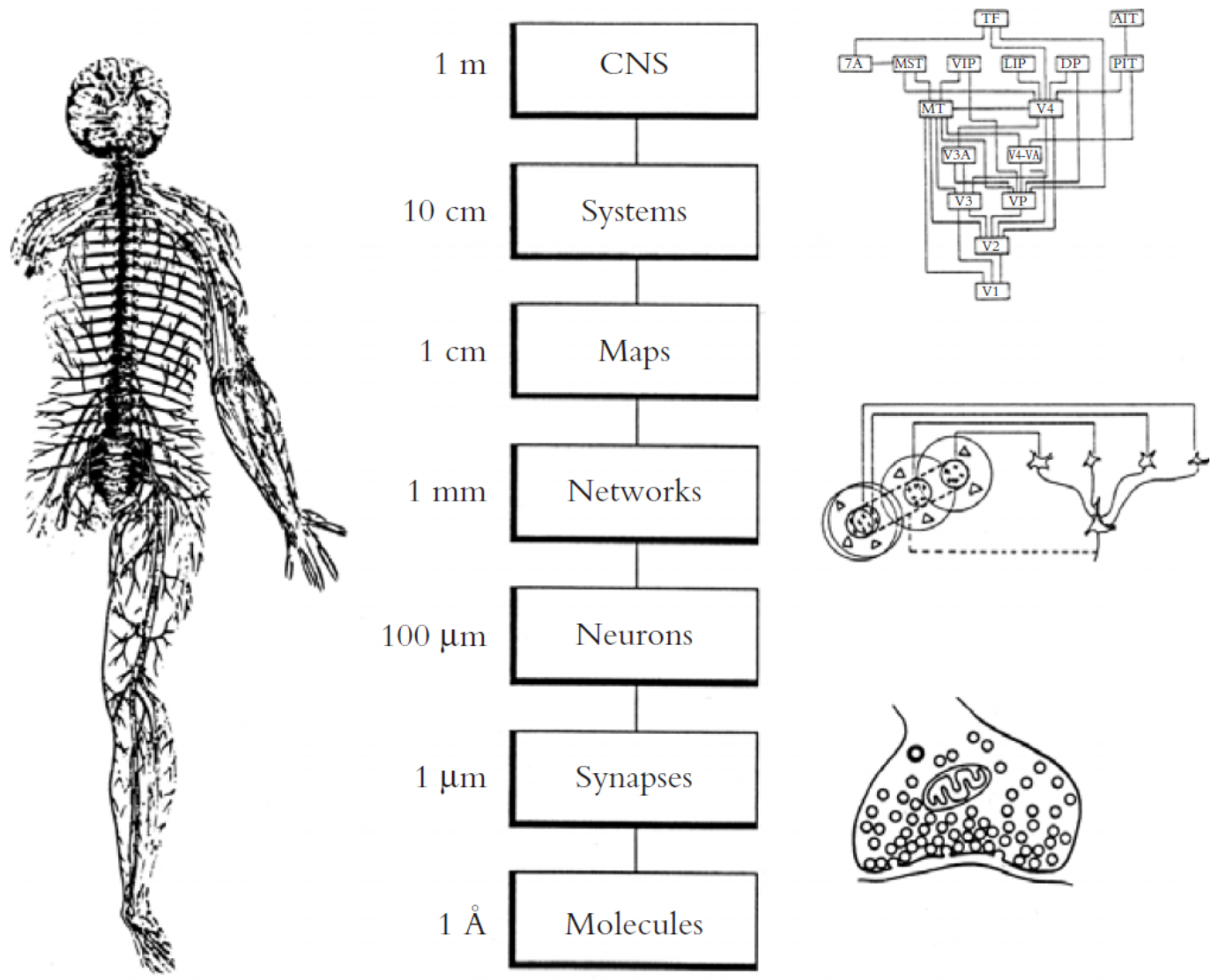

FIGURE 2.6: Levels of organization within human nervous system Churchland and Sejnowski's classic diagram of levels in neuroscience. Adapted from (Craver, 2007, fig. 5.5).

used in explanatory and descriptive analyses of the phenomenon; they define the focus of the analysis with the appropriate language used in it. Figure 2.7 (adapted from Franklin, Strain, Snaider, McCall, \& Faghihi, 2012, fig. 4) gives an example in the study of cognitive processes in different fields of science.

With the theoretical foundation laid so far, in Chapter 3, the neurocognitive theories of consciousness are reviewed, and the global workspace theory is presented. 


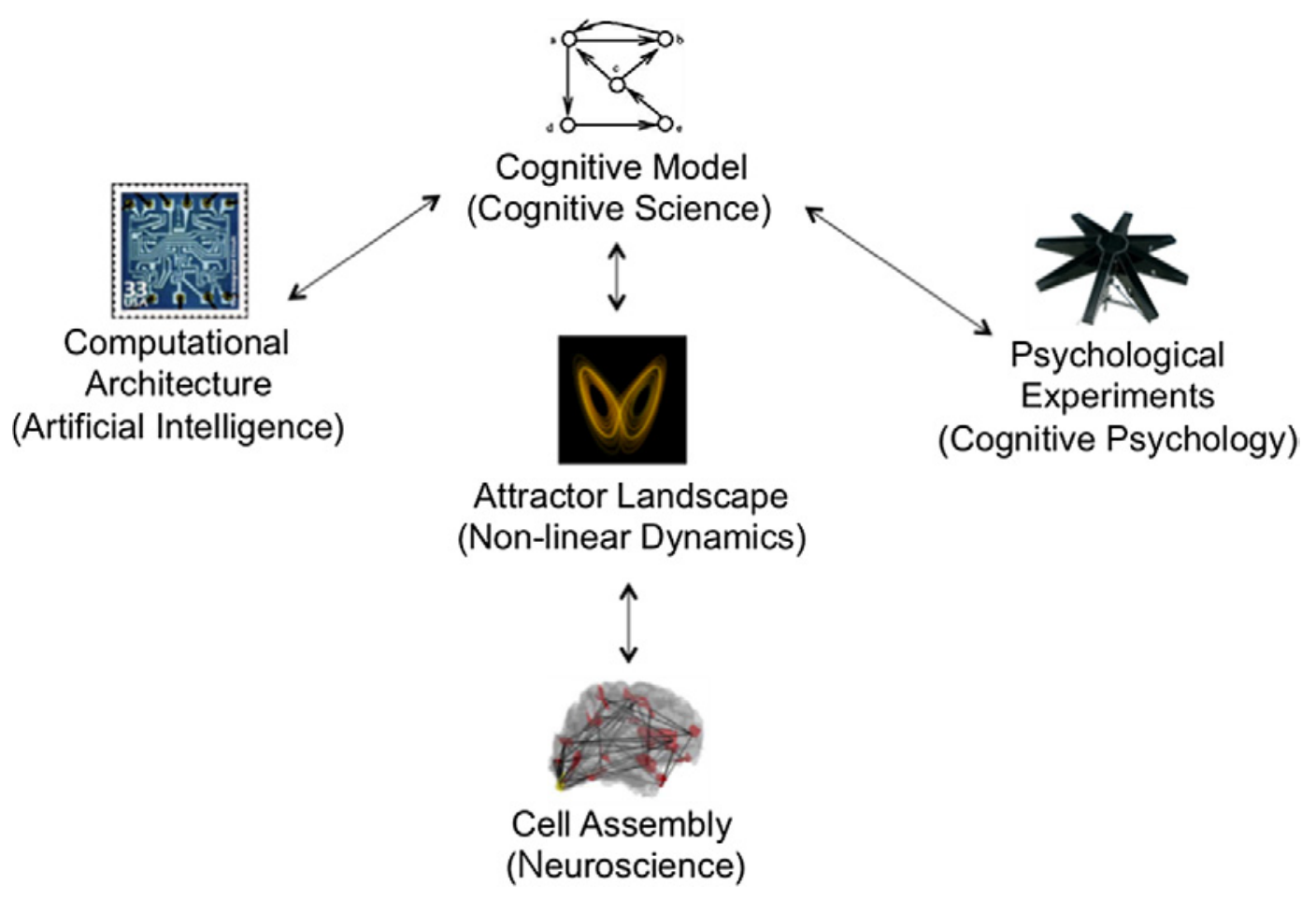

FIGURE 2.7: Levels of abstraction in the study of brain and cognition Adapted from (Franklin et al., 2012, fig. 4). 



\title{
Chapter 3
}

\section{Neurocognitive Theories of Consciousness}

\author{
Last night, I took my difficulty to the Elder of the Magians; \\ Who, by strengthening of sight, the solving of conundrums made. \\ — Ḥִ̂̄FEZ OF ShĪrĀZ (CA. 1315-1390)
}

Chapter 1 discussed the philosophical notions of consciousness and introduced the questions that had engrossed the philosophy of mind. The study of consciousness, however, is a multidisciplinary field of research wherein armchair philosophy fails to iron out all the conundrums singlehandedly; no matter what stance we take towards consciousness (eliminative materialism, substance dualism, or anything in between ${ }^{1}$ ), we ultimately need to answer the question of how "the mental" interacts with the physical world. The nervous system (more specifically, the brain) is the closest physical entity to the mind. Hence, any account of consciousness must establish (or at least, outline) links between the philosophical aspects of consciousness and the neural substrate within (or upon) which it is hosted.

Efforts have been made to bridge between the two disciplines of philosophy (more specifically, what Mandik (2017) calls naturalized philosophy) and neuroscience. Most notably, in her Neurophilosophy, Patricia Churchland (1989) attempted to introduce the philosophy of mind to neuroscientists and neuroscience to philosophers (Mandik, 2017). However, most of the contributions to the literature of neurophilosophy are made by philosophers, hence its dominant taste of philosophy. As Mandik notes, neurophilosophy have mainly put three questions in the spotlight: the question of state consciousness (what makes a state conscious?); the question of transitive consciousness (what is the subject of consciousness when one is conscious of it?); and the question of phenomenal character ("when one has a conscious state, in what consists the properties in virtue of which there is something it's like for one to be

\footnotetext{
${ }^{1}$ Details of various theoretical accounts towards consciousness are beyond the scope of this dissertation and will not be discussed any further. The two accounts mentioned can only be loosely considered as extreme opposites in the vast "spectrum" of theories of consciousness - if one can call it a spectrum.
} 
in that state?" Mandik, 2017, p. 460). These questions are referred to as $Q_{\text {state }}$, $Q_{\text {transitive }}$, and $Q_{\text {phenomenal }}$ in this dissertation, respectively.

It can be noticed that the study of consciousness among scientists lacks the philosophical accuracy exercised by their philosopher peers. In return, the scientific literature on consciousness is more pragmatic and addresses less abstract problems; more specifically, $Q_{\text {state }}$ and $Q_{\text {phenomenal }}$ have received the most attention among neuroscientists. The former has mainly been studied in the research into neural correlates of consciousness (NCCs) while the latter has been the subject matter of models of consciousness. This chapter reviews the NCC research. In Section 3.2, the global workspace theory of consciousness and its backbone - namely, the global access hypothesis - are reviewed by the relevant body of evidence favoring them. In the same section, Dennett's multiple drafts model is reviewed and compared with the GWT. Finally, in Section 3.3, global workspace models are discussed. This chapter is a foundation for Chapter 4 , which is about the computational implementations of these models.

\subsection{From Neural Correlates to Models of Consciousness}

David Chalmers (2010, Chapter 2) has laid down an agenda for the scientific study of consciousness. In his opinion, a scientific account of consciousness needs to, ultimately, relate first-person data of subjective experience to third-person, objective data reflected in observable behaviors or neural processes. He distinguishes six projects for a proper science of consciousness: (1) establishing solid explanations ${ }^{2}$ of third-person data; (2) contrasting conscious and unconscious processes; (3) probing the contents of consciousness; (4) finding neural correlates of consciousness; (5) systemizing the connections between first- and third-person data; and (6) inferring fundamental principles of consciousness (2010, pp. 41-47). His proposal mainly concerns answering the descriptive what-questions while leaving aside the explanatory how-questions and the functional why-questions (cf. Chapter 1).

$\mathrm{He}$, at least implicitly, hopes that having all pieces of the puzzle in place yields an adequate scientific understanding of consciousness; the answers to the howquestions, for instance, will be revealed if we succeed in stringing the shreds of conscious phenomena (his first five projects) and inferring the constitutional rules of consciousness within this constellation. Some might not find this approach promising - if ever feasible. Nonetheless, it helps us understand why the study of NCCs has become a vital element in the neuroscientific study of consciousness.

NCCs are the neural system(s) or assemblies that can be associated with different aspects of consciousness, e.g., conscious mental states or their contents. This definition posits that NCCs exist somewhere in the brain, and the quest is to

\footnotetext{
${ }^{2}$ It is worth mentioning that, based on how Chalmers frames the project (2010, pp. 4142 ), it is likely that mere description or explication - not necessarily explanation - of the thirdperson data would suffice the goal of his first project. See Section 2.1 for the distinction between description/explication and explanation.
} 
find and characterize them. The ultimate goal in the search for NCCs is to use them in the explanations of consciousness, and if that is a viable option, somehow reduce consciousness to its neural correlates. Before doing so, one has to clarify what she means by consciousness, and characterize the association that would count as "correlation." There are several conceptual and methodological obstacles in characterizing NCCs (and making inferences upon them) which are beyond the scope of this chapter - there are entire books on the matter, e.g., see (Metzinger, 2000). One of the most critical conceptual issues - after defining what does (and does not) count as correlation - is that mere correlations can hardly provide any solid explanation of the origins of the association (Koch, Massimini, Boly, \& Tononi, 2016; Rees \& Frith, 2017).

Neural correlates can be defined in (at least) three different ways, based on the notion of consciousness in question. For state consciousness $\left(\mathrm{NCC}_{\text {state }}\right.$; in line with $\left.Q_{\text {state }}\right)$ it can be defined as follows:

A neural system $N$ is an NCC if the state of $N$ correlates directly with states of consciousness. (Chalmers, 2010, p. 61)

On the other hand, NCCs of contents of consciousness $\left(\mathrm{NCC}_{\text {content }}\right.$; addressing, loosely speaking, $Q_{\text {transitive }}$ ) can be defined as:

A neural correlate of the contents of consciousness is a neural representational system $N$ such that representation of a content in $N$ directly correlates with representation of that content in consciousness. (Chalmers, 2010, p. 65)

It is far trickier to answer $Q_{\text {phenomenal }}$ through the study of the neural systems associated with the phenomenal properties of consciousness $\left(\mathrm{NCC}_{\text {phenomenal }}\right)$. An $\mathrm{NCC}_{\text {phenomenal }}$ can be defined as:

A state $N_{1}$ of system $N$ is a neural correlate of phenomenal property $P$ if $N$ 's being in $N_{1}$ directly correlates with the subject having $P$. (Chalmers, 2010, p. 68)

Although NCC research has received much attention, it is crucial to not forget what has made it relevant to consciousness research in the first place: its promise of helping the scientific endeavors in bridging the gap between the first- and third-person data. Moreover, it has a (somewhat) strong premise: that the (phenomenal) aspects of consciousness can be reduced to the candidate NCCs. However, it seems NCC research has been more successful in describing the associations between aspects of consciousness and neural phenomena than adequately explaining them. This brings us to models of consciousness, which encompass NCCs within more comprehensive frameworks.

A model of consciousness is a theoretical description of how brain properties relate to phenomenal properties of consciousness using a mechanistic explanation (Seth, 2007). The last part of the definition distinguishes models of consciousness from theories of consciousness as the latter do not necessarily commit to establishing 
a mechanistic understanding of consciousness (Seth, 2007). The term "models of consciousness" has been used slightly differently in the literature of machine consciousness with less commitment to the realization of the model in a neural substrate. For instance, Reggia (2013) deems higher-order thought (HOT) theories (Rosenthal, 1996, 2005; also cf. Carruthers, 2016) as models of consciousness since they provide a mechanistic explanation of consciousness and can be realized computationally. Conversely, Seth (2007) opposes HOT theories as such since he believes such mechanistic explanation should be anchored to a neural realization in order to count as a model of consciousness. On the other hand, although Seth (2007) includes Dennett's (1993) multiple drafts model among models of consciousness, it is left out in (Reggia, 2013), perhaps because it is not clear how this model can be explicitly implemented computationally.

Among the various models of consciousness (cf., e.g., Reggia, 2013; Seth, 2007), two are of greater importance for the subject of this thesis: the global workspace models (GWMs), and the multiple drafts model (MDM). GWMs are discussed in details in the next section and the MDM will be briefly introduced accordingly.

\subsection{The Global Workspace Theory of Consciousness}

The global workspace theory (GWT), first presented by Bernard Baars in his magnum opus A Cognitive Theory of Consciousness (1988), initially aimed at contrasting conscious processes from the unconscious ones. It later matured into a comprehensive cognitive theory of consciousness (Baars, 2002) as it received empirical support (Franklin et al., 2012). It is crucial to note that Baars' original (1988) proposal for the GWT is a functional theory of consciousness, yet it became a stepping stone for the later models of phenomenal consciousness. The GWT assumes that the unconscious (background) processes run in parallel in the distributed system of (specialized) processors and it is the "coalitions of these processes [that] enable an agent ... to make sense of the sensory data coming from the current environmental situation." (Franklin, 2011, p. 328) Moreover, since numerous processes are simultaneously running in the brain at any given time, different coalitions emerge in the brain, and, as Franklin puts it,

[These] coalitions, incorporating the results of the processing of sensory data, compete for attention in what Baars calls a global workspace. The contents of the winning coalition are broadcast to all other processes. The contents of this broadcast are proposed to be phenomenally conscious. This conscious broadcast serves to recruit other, unconscious, processes to be used to select an action in response to the current situation. GWT is therefore a theory of how consciousness functions within cognition. (2011, p. 328)

In this theory, the global workspace $(\mathrm{GW})$ acts both as a bottleneck ${ }^{3}$ of information

\footnotetext{
${ }^{3}$ Note that the term bottleneck has a different connotation when used in a broader context of (computational) resources, wherein it is often used as a metaphor of how limited resources lead to
} 


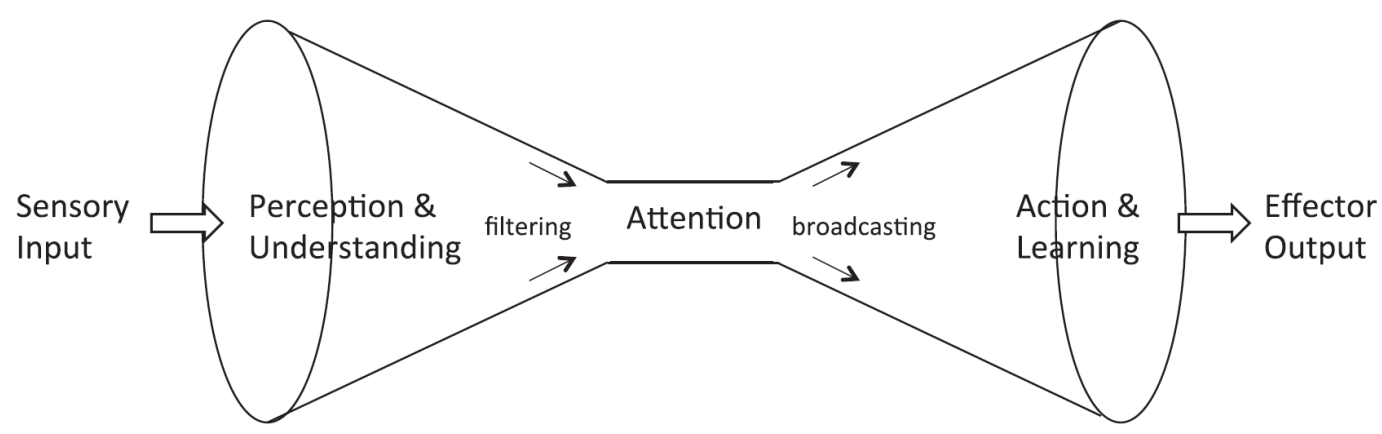

FIGURE 3.1: Global workspace as a bottleneck and as a broadcaster Information from various processors (e.g., brain's sensory regions) enter the funnel (left) and compete to get through the bottleneck of attention (i.e., GW; middle). Those winning the competition are broadcast to other processors (right). Adapted from

(Franklin et al., 2016, fig. 1).

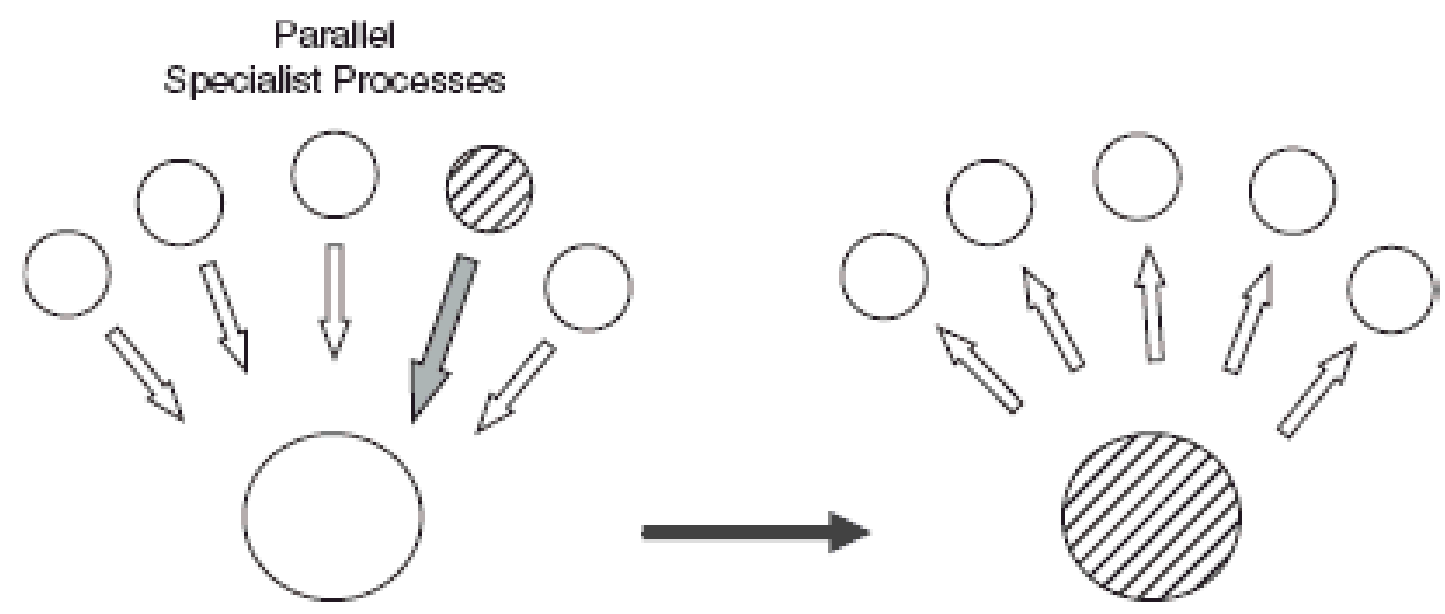

Global Workspace

FiguRE 3.2: Two aspects of the global workspace as a stage Left: Parallel specialized processors try to dominate the global workspace. Right: When information enters the global workspace it is broadcast to all processors. Adapted from

(Shanahan, 2010, fig. 4.1).

and a broadcaster. Figure 3.1 (Franklin et al., 2016, fig. 1) roughly shows how GW filters the competing information "produced" by different sensory coalitions and broadcasts to other areas. It can be generalized to other processes as well. See Figure 3.2 (Shanahan, 2010, fig. 4.1). Before discussing the details of how GWT is realized in the brain, we need to discuss the theoretical and empirical foundations of the GWT, to which Sections 3.2.1 and 3.2.2 are dedicated.

prioritizing the processes. This prioritization limits the performance of the system as a whole since not all processes can "path through" that bottleneck at the same time. 


\subsubsection{The conscious limitedness and the unconscious immenseness}

The point of departure for the GWT is the well-established finding that limited conscious capacity of the brain (e.g., in coherent binding of sensory inputs, conscious access to memory, and cognitively demanding tasks) coexists along with many unconscious, parallel processes (such as coordination of body movements and maintaining balance, or perception of volumes in vision) happening within a "massive beehive of neural assemblies, cells, layers, and connections, each flexibly specialized in some set of tasks." (Baars, 2017, pp. 232-233). The limited conscious capacity has been known to philosophers, psychologists, and cognitive (neuro)scientists for decades. For a detailed review of limited capacity in attention, action, memory, and vision, see (Baars, 1988, secs. 1.3.3-1.3.6). Baars also reviews the consensus around unconscious parallel processes in the brain and discusses their properties (Baars, 1988, sec. 1.4). For a more concise review, see (Baars, 1997b). The GWT suggests the the global access hypothesis $(\mathrm{GAH})$ can explain how these different kinds of processes coexistand interact. Here, two of the most intuitive psychological bodies of evidence for parallel processes are reviewed: the mental lexical access, and the autobiographical memory.

It is estimated that an average 20-year-old native English speaker knows roughly 42,000 unique lemmas (Brysbaert, Stevens, Mandera, \& Keuleers, 2016). Many of these lemmas are shared by different words, meaning that the native speakers' lexicon is far more abundant. Moreover, many words with the same morphology differ remarkably in their meanings. It is evident that one does not - and, in fact, cannot - consciously retrieve meanings of every word from her lexicon to comprehend sentences in the everyday use of language. Additionally, there is compelling evidence that the semantic context of words is autonomously (and unconsciously) processed before the correct connotation of the word is (consciously) comprehended (Swinney, 1979; as cited in Baars, 1988). Baars elaborates on this with an example:

A typical experiment in this literature has the following format: Subjects listen to a sentence fragment ending in an ambiguous word, such as "They all rose ..." The word "rose" can be either a verb or a noun, but in this sentence context it must be a verb. How long will it take for this fact to influence the interpretation of the next word? To test this, one of two words is presented, either flower, or stood. Subjects are asked to decide quickly whether the presented word is a real English word or not. If the subjects make use of the sentence context in their lexical decision task, the verb "rose" should speed decisions for "stood," because the two words are similar in meaning and syntax; if the context is not used, there should be no time difference between the verb "stood" and the noun "flower." Several investigators have found that for the first few hundred milliseconds, the sentence context has no influence at all ... Thus it seems as if lexical access is autonomous and context-free for a few hundred milliseconds. After this period, prior context does influence the choice of interpretation. (1988, p. 46) 
More recent research has focused on characterizing the neural processes that give rise to this phenomenon (for recent studies on semantic priming, cf., e.g., Fahimi Hnazaee, Khachatryan, \& Van Hulle, 2018; Lerner, Bentin, \& Shriki, 2012).

Another interesting phenomenon is the quick accessibility of individual memories within the autobiographical memory. In his classic study, Standing (1973) reports that, under certain measurement conditions, subjects are capable of distinguishing as many as 10,000 images previously seen only for a few seconds each. Based on these findings, Baars writes:

We can get an everyday sense of this remarkable memory performance from the common experience of recognizing a film seen only once, many years ago, with a sudden sense of familiarity. Often we can even predict the next scene. It seems that the brain creates memories of the stream of experience merely by paying attention to it; but humans are always paying attention to new and interesting things, suggesting that our spontaneous autobiographical memory must be very large indeed. Once again we have a vast unconscious domain, and we gain access to it using conscious thought. Mere consciousness of an event helps to store it in memory, and when we experience it again we can voluntarily recognize one distinctive episode from many millions in memory. (2017, p. 234)

\subsubsection{The Global Access Hypothesis}

The GWT introduces GAH using the classic theater metaphor of consciousness (Baars, 1997a). In this metaphor, parallel processes compete in order to take over the stage, and the contents of the winner become accessible (or, is "broadcast") to other processes. The working memory is also on the stage; it can be seen as either as a computational resource "within" the GW, available to the winning processes, or as a "medium" hosting the information contents of those processes. ${ }^{4}$ The content appearing on the stage is experienced consciously by the subject when illuminated by the spotlight of attention.

Figure 3.3 (adapted from Baars, 2017, fig. 16.1) shows a schematic view of GAH and the stage, the spotlight, and the processes accessing the GW. These processes, both the competitors and the audience - although most of them can undertake both roles - are not limited to sensory inputs and verbal and non-verbal outputs; they can also be related to perceptual and cognitive contexts (examples of the latter are intentions, expectations, and the "self") or the unconscious resources (such as interpretations, memories, language, and automatism).

The GAH has received a staggering amount of converging empirical and philosophical support (cf., e.g., Cooney \& Gazzaniga, 2003; Dehaene, Kerszberg, \& Changeux, 2001; Dehaene, Sergent, \& Changeux, 2003; Dennett, 1993; Freeman, 2003; all as cited in Baars, 2017). Although there are discrepancies in characterization and

\footnotetext{
${ }^{4}$ It seems that Baars leans toward the latter account wherein the working memory and the GW are not totally distinct entities - implying that they are necessarily the same thing. See (Haikonen, 2011) and the discussion on Shanahan's model in Secion 3.3.
} 


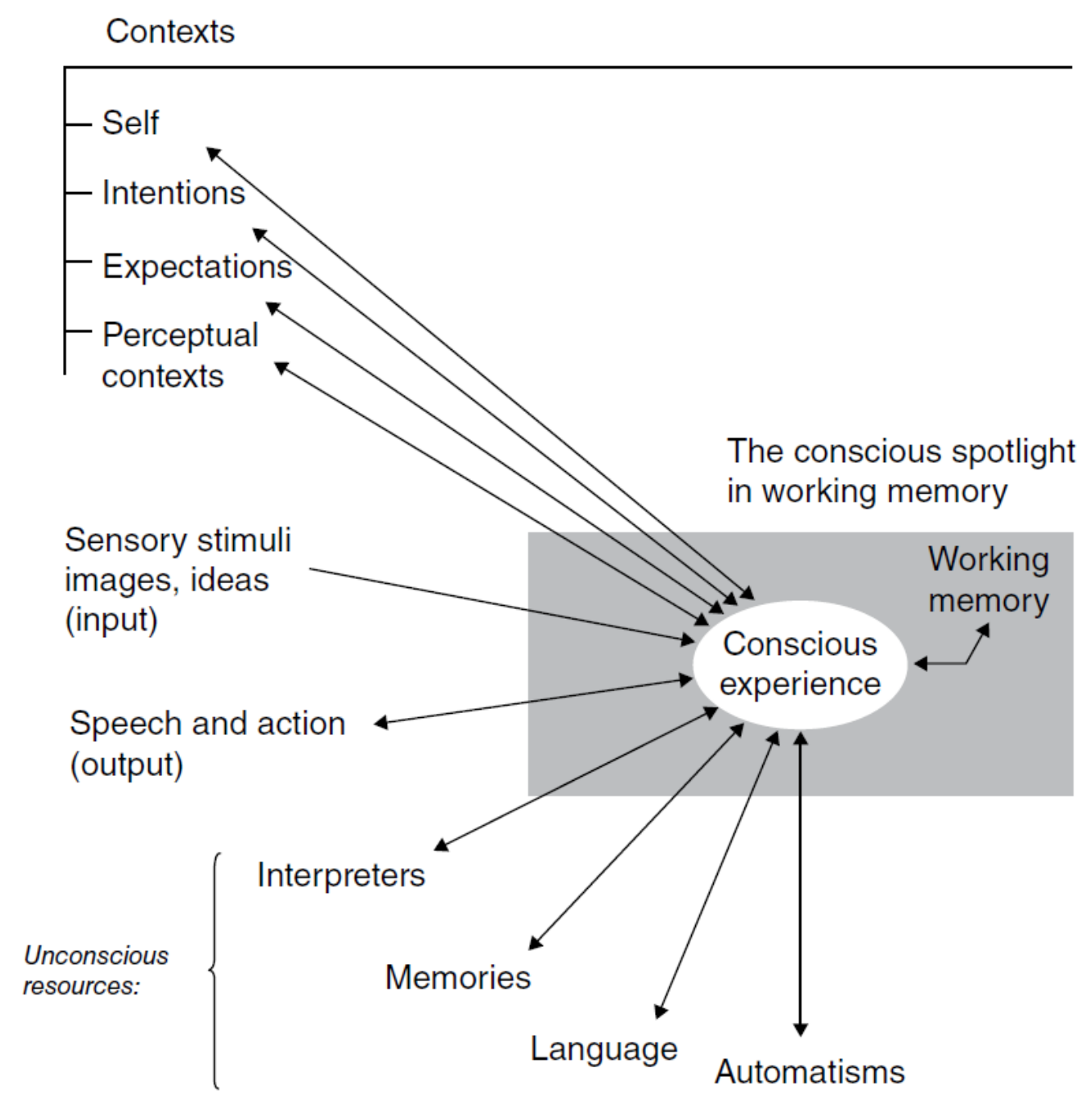

FIGURE 3.3: . Schematic diagram of global access Various processes (contextual, sensory inputs, and verbal/non-verbal outputs) and unconscious resources have access to working memory (i.e., the global workspace according to Baars), and the conscious experience is where the spotlight of attention illuminates the global workspace. Adapted from (Baars, 2017, fig. 16.1). 
properties of the "GW" in these theories, there is a decent consensus among scholars around GAH, especially with formalizations close to that of GWT - and such consensuses are quite scarce in the field of consciousness studies. A more detailed review of the literature (from 1983 to 2001) supporting GAH can be found in (Baars, 2002). Before moving on to GWMs (which are realizations of GWT) let us address Daniel Dennett's multiple drafts model as it gives additional insights into the GAH.

\subsubsection{Multiple Drafts Model}

Daniel Dennett's MDM (Dennett, 1993, 2001) belongs to (and stands out as an exemplary member of) the family of narrative interpretive theories of consciousness. It was developed as an alternative to the metaphor of "Cartesian Theater." The Cartesian Theater metaphor posits that consciousness is a result of the consolidation of information at some spatial or functional location or in a special mode or format wherein they become accessible to the "self." (Van Gulick, 2018, sec. 9.4). Discussion of Descartes' dualist theory of mind is well beyond this manuscript, but the keen reader can consult (Hatfield, 2018, sec. 3.4; Robinson, 2017, sec. 1.2; Van Gulick, 2018 , sec. $4.5 \& 8.1$ ).

The MDM borrows "elements of both representationalism and higher-order theory but does so in a way that varies interestingly from the more standard versions of either providing a more interpretational and less strongly realist view of consciousness." (Van Gulick, 2018, sec. 9.4, par. 1) In the MDM, multiple pieces of information made by brain regions are considered "drafts", passed from one region to another and subject to constant modification as they travel around. Drafts gain "fame" in the brain based on the degree to which they engage the processors. The drafts compete to gain more fame, and content of the most famous draft becomes "conscious." According to MDM, qualia are not phenomena that need explanations but mere epiphenomena of gaining the most fame. Hence, the MDM is an eliminativist theory of consciousness (Ramsey, 2019, sec. 3.3). This implies that many of the questions mentioned in Chapter 1 become irrelevant - what does it mean to explain something that does not "exist"? The MDM also explains the self as a "center of narrative gravity" (Dennett, 1992). As Van Gulick writes,

The MDM treats the self as an emergent ${ }^{5}$ or virtual aspect of the coherent roughly serially narrative that is constructed through the interactive play of contents in the system. Many of those contents are bound together at the intentional level as perceptions or fixations from a relatively unified and temporally extended point of view, i.e., they cohere in their contents as if they were the experiences of [an] ongoing self. But it is the order of dependence that is crucial to the MDM account. The relevant contents are not unified because they are all observed by a single self, but just the converse. It is because they are unified and coherent at the level of

\footnotetext{
${ }^{5}$ Van Gulick's use of the term "emergent" is not very accurate; in the MDM, the self is merely an imaginary abstraction of the narrative formed in the brain, and Dennett tends to distance himself from emergence.
} 
content that they count as the experiences of a single self, at least of a single virtual self. (2018, sec. 9.4, para. 3)

The MDM has been extremely controversial yet amazingly influential. One of the most important objections against the MDM - and more specifically, the way Dennett sells it in his seminal book Consciousness Explained (1993) - is that it, although built upon empirical evidence, fails to provide a realist, scientifically testable ${ }^{6}$ model for consciousness (see, e.g., McGinn, 1995). The keen reader will gain insights into the critiques of the MDM in the peer commentaries of Dennett and Kinsbourne's (1992) paper. The MDM and the GWT both endorse the GAH, but in different ways.

The MDM is in line with the GAH, as it emphasizes on the accessibility of the drafts to the processors, but does not set forth any locus where they consolidatemore precisely, it explicitly denies that. The GWT, on the other hand, limits the global accessibility to the content reaching the stage of the GW, while there is no stage in the MDM metaphor. The GWT (even its original version where it has no commitment to any physical realization, see next subsection) is more "scientific" for at least three reasons: it can be formally defined, beyond metaphorical interpretations; it gives a mechanistic explanation for consciousness; and, most importantly, it makes specific predictions that can be verified scientifically. The GWMs are even more powerful compared to the basic GWT for they address realization of the GW, and, one way or another, anchor themselves to the underlying neurobiology and neuroanatomy of the brain. These realizations are discussed in the next section.

\subsection{Global Workspace Models: The Realizations of the GWT}

As mentioned earlier in this chapter, Baars' (1988) basic GWT is a functional theory of conscious experience - it does not commit to any (specific) realization of its architecture. This basic theory makes six theoretical predictions, as mentioned in (Baars, 2017, table 16.1). Based upon them, he proposes seven models with increasingly wider scopes of empirical predictions (for a short overview, see Baars,

\footnotetext{
${ }^{6}$ It is quite interesting that Dennett, rather humbly, concludes Consciousness Explained with the following paragraph:

My explanation of consciousness is far from complete. One might even say that it was just a beginning, but it is a beginning, because it breaks the spell of the enchanted circle of ideas that made explaining consciousness seem impossible. I haven't replaced a metaphorical theory, the Cartesian Theater, with a nonmetaphorical ("literal, scientific") theory. All I have done, really, is to replace one family of metaphors and images with another, trading in the Theater, the Witness, the Central Meaner, the Figment, for Software, Virtual Machines, Multiple Drafts, a Pandemonium of Homunculi. It's just a war of metaphors, you say-but metaphors are not "just" metaphors; metaphors are tools of thought. No one can think of consciousness without them, so it is important to equip yourself with the best tools available. Look what we have built with our tools. Could you have imagined it without them? (Dennett, 1993, p. 455)
} 
2017, table 16.2). Although these models are in accord with specific empirical evidence from the neuroscientific study of consciousness, they are not specifically (or visibly enough) linked to plausible neuroanatomical realizations.

Further research tried to bridge the gap between the mental and the realization levels of the mechanistic explanation of consciousness. For a comprehensive review, see (Baars, 2002). Among the GWMs studied in the literature, Murray Shanahan's (2010) anatomically-inspired version of a global neural workspace model (referred to hereafter by SGWM) deserves more attention as it tries to incorporate embodiment within a GW framework. This model roots in his earlier architecture (Shanahan, 2006) that explicitly included the internal stimuli in a GWM. ${ }^{7}$ In the remainder of this chapter, SGWM is discussed.

Shanahan augments an intermediate level of abstraction, namely, neural dynamics, between the TLMs' two lowest levels of analysis (viz., $L_{\text {mental }}$ and $L_{\text {realization; }}$; see Chapter 2). Consequently, SGWN puts forth an explication for the (possible) structure of the GW, and an explanation for how this structure serves as a GW. Haikonen (2011) compares various aspects of Baars' GWMs to Shanahan's SGWM. Perhaps one of the most important differences between them is that Shanahan emphasizes that GW is an infrastructure of information exchange (wherein competition takes place as well). Hence it is substantially different from Baars' working memory account. Haikonen elaborates on this distinction:

Shanahan's view about the global workspace is different. Firstly, he wishes to distance himself from the theater model by recasting the role of the global workspace. Instead of a working memory, the global workspace should be thought as a communications infrastructure that connects the various autonomous units with each other: "Indeed, unless recast this way, the putative workspace might be mistaken for a dedicated brain region, something akin to the Cartesian theater ridiculed by Dennett, [a place in the brain where "it all comes together and consciousness happens.']" [(Shanahan, 2010, p. 111)] Shanahan proposes that in the brain, the global workspace consists of the white matter connective core that connects the various brain areas with each other. But this infrastructure is not to be taken only as a transmission medium. According to Shanahan: "GWS is not only the locus of broadcast, but also the arena for competition between rival coalitions and the medium of coupling for the members of those coalitions." [(Shanahan, 2010, p. 147)] This infrastructure has limited capacity and therefore forms a temporally limiting bottleneck; this would explain the serial nature of consciousness. (Haikonen, 2011, pp. 339-340)

\footnotetext{
${ }^{7}$ SGWM, has attracted more attention from philosophers compared to its peers, e.g., Dehaene et al.'s (2003) neural global workspace or the network-theoretic GWM of Wallace and Glazerbrook (Glazebrook \& Wallace, 2009; Wallace, 2005). Hence, and for the sake of brevity of the thesis and its focus on MC, only the original GWT and SGWT are discussed in this manuscript. Nevertheless, the keen reader is strongly encouraged to follow the references and study other GWMs as well (e.g., see Seth, 2007, Section 2).
} 
Shanahan uses graph theory to characterize possible structures that can be plausible candidates for a putative GW. He seeks a network structure that has two properties: it has specialized autonomous modules, and the modules are efficiently interconnected. Such networks are called small-world structures (SWS) ${ }^{8}$ as they share similar mathematical properties with other networks found in the wild ${ }^{9}$. SWSs entered the scientific discourse after Watts and Strogatz (1998) introduced a rewiring algorithm that recursively optimizes the structural properties of a regular graph to eventually manifest properties of $\mathrm{SWSs}^{10}$. SWSs has been studied widely since, and a rich literature asserts that brain networks have small-world properties (SWPs; Bassett \& Bullmore, 2006; Sporns \& Zwi, 2004; for a more systematic introduction, consult Sporns, 2010, Chapters 3-4). Several rewiring algorithms have been proposed that resemble the natural development of the human brain and can generate SWSs (e.g., see Jarman, Steur, Trengove, Tyukin, \& van Leeuwen, 2017).

Shanahan (2010, Chapter 4) explains in two steps how the coalition can lead to a GW-like structure suitable for the broadcast if information. First, he investigates formal representations of graphs that have the desired structure. He starts with the classic Watts and Strogatz network and suggests an adapted graph wherein highly intraconnected communities enhance the modularity of the network (pp. 119-121). He then distinguishes connector hubs, i.e., the nodes that with high trans-modular connectivity, and names the links between such hubs the connective core (pp. 121-122). Finally, he suggests that the communities themselves can be thought of as smaller SWSs. Hence, he generalizes the network into a scale-free ${ }^{11}$, hierarchical structure, wherein modularity and SWPs are present at different levels of organization-locally and globally (p. 122). These models are shown in Figure 3.4 (adapted from Shanahan, 2010, figs. 4.6-4.8).

In the second step, he proposes candidate neuroanatomical structures for a presumed GW. More specifically, he suggests that the long-range white matter tracts (connecting distant regions of the cortex) can constitute a substrate for the GW, and the "thalamocortical pathways [that] relay traffic to and from different parts of the cortex, and may also play a role in the putative workspace." (Shanahan, 2010, pp. 125-126) Then, he shows that applying the hierarchical SWS introduced in the first step to structural parcellation of the cortex into highly intraconnected brain regions (as in, e.g., Hagmann et al., 2008) yields a network structure similar to that of the first step (p. 126). Finally, he distinguishes a connective core within the anatomical network, and concludes that it is indeed the possible locus for a neural GW in the brain. This proposal is backed by neuroanatomical findings and mathematical modeling of such network structures - and most importantly, the connective core is

\footnotetext{
${ }^{8}$ Or small-world networks (SWNs).

${ }^{9}$ Most important characteristics of SWSs are high clustering coefficient (which is an indicator of modularity, thanks to highly intraconnected modules) and low average path length between any nodes of the network (thanks to the long-range intermodular connections).

${ }^{10}$ For a better understanding of SWSs and their applications in neuroanatomy, see (Sporns, 2010, Chapters 1-2).

${ }^{11}$ Scale-free networks show similar modularities/SWPs across levels. See (Lee et al., 2010) for scale-free networks in the brain.
} 

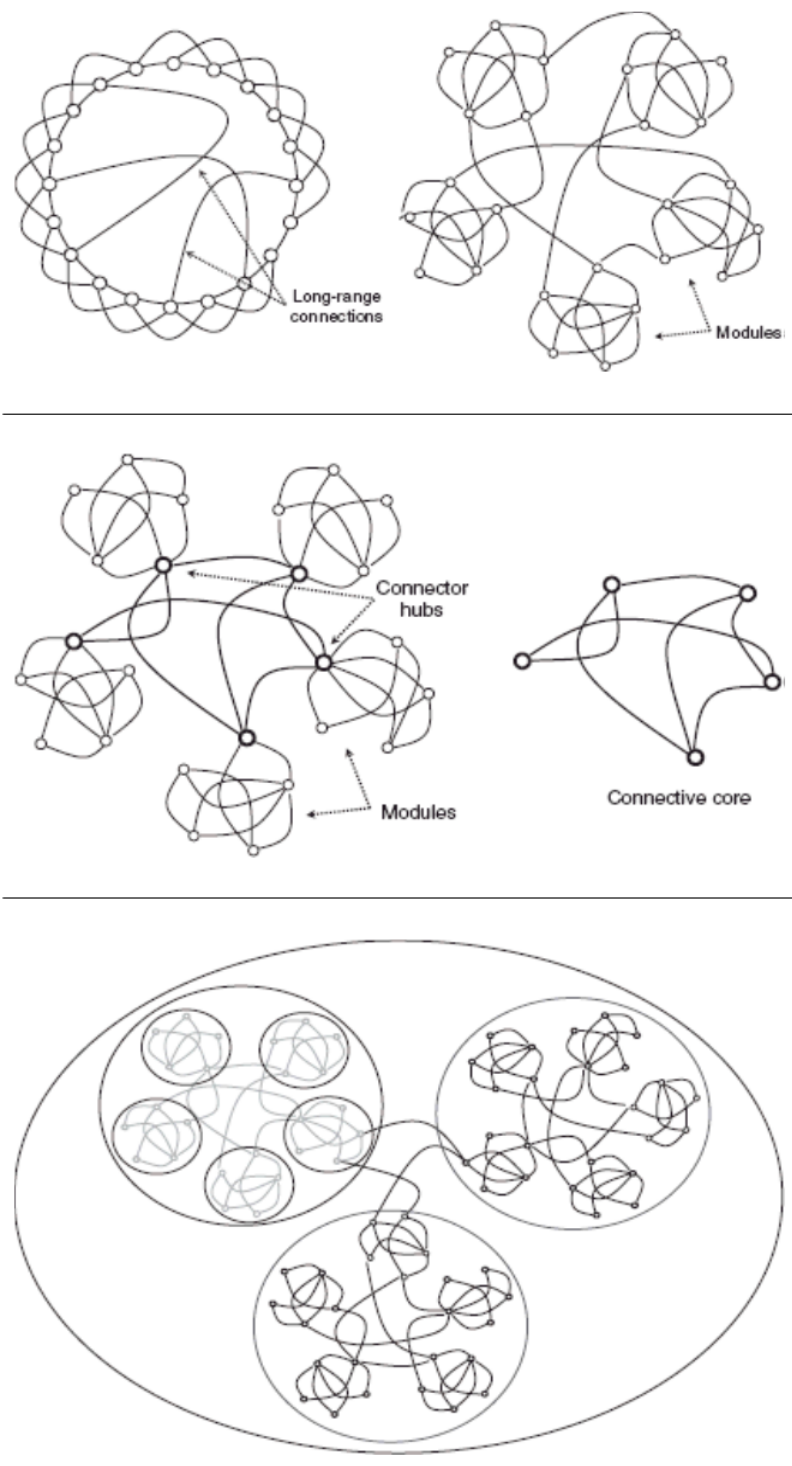

FIGURE 3.4: Small-world structures in graph theory Top: Two kinds of SWN Left: Watts-Strogatz network, wherein short-range edges of a regular graph are replaced by long-range links, providing shortcuts across the lattice hence reducing the average path length without significantly reducing the clustering coefficient. Right: a modular SWN. A number of densely intra-connected modules connected to each other with a sparse set of inter-module links. Middle: A modular SWN with connector hubs (outlined in bold) which are nodes with high trans-modular connectivity. The "connective core" is the connector hubs plus the arcs joining them. Down: An SWN with three levels of hierarchical modular organization where ellipses emphasize the hierarchical structure. Adapted from (Shanahan, 2010, figs. 4.6-4.8). 
capable of manifesting the principal properties of a GW (see Figure 3.1). Shanahan writes,

The connective core of a hierarchically modular network is topologically well placed to realize both broadcast (because influence funnels into and fans out from the centre) and competition (because it acts as a limited capacity bottleneck). So it is an ideal candidate for the anatomical locus of a global workspace. (2010, fig. 4.11 caption)

These models are depicted in Figure 3.5 (adapted from Shanahan, 2010, figs. 4.94.11).

Shanahan's SGWM is of the highest value among GWMs for two main reasons. Firstly, it has more explanatory power compared to its predecessors; e.g., it explains embodiment and explicates the inner narrative property of consciousness. Secondly, and most importantly, it is decently tethered to the neural substrate via the intermediate level of abstraction it places between $L_{\text {mental }}$ and $L_{\text {realization }}$. The latter point has a remarkable implication - it guarantees a mechanistic link between those levels of analysis, hence partitioning the arduous enigma of answering the how-questions into two smaller, more feasible problems: explanations at $L_{\text {mental }}$, and explications of (structural) realization of the $\mathrm{GW}$ in the $L_{\text {realization }}$.

\subsection{A Closer Look at the GWT}

The GWT is a promising foundation upon which cognitive models of consciousness can be erected. It is a functional framework that makes reasonable explanations addressing $Q_{\text {state }}$ - and $Q_{\text {transitive }}$ in part (e.g., Dehaene \& Naccache, 2001). It also makes behavioral predictions based on its cognitive architecture (cf. references of Sections 3.2.1 and 3.2.2). Furthermore, SGWM adds a missing piece to the puzzle by connecting the behavioral outcomes to neuroanatomy. Albeit the success of GWT and SGWM, they do not address $Q_{\text {phenomenal }}$. Additionally - and more importantlythey seem to miss the questions they were meant to ask. Before concluding this chapter, let us review these two issues, starting with the latter.

\subsubsection{Addressing nihil ad rem $^{12}$ : What was the question being answered?}

In a commentary on Baars' (1997a, 1997b) GWT, Elitzur (1997) raises a point: What is the actual question that Baars' theory is trying to answer? He tells a Jewish joke of "the lunatic scholar who runs in the streets in search for a good question to his answer," (p. 320) and accuses Baars of answering the questions that have not been asked in the first place - or, as in a Persian proverb, shaving an unowned head. More specifically, he, like Combs (1997), argues that Baars' contrastive analysis of conscious and unconscious processes is turning the questions upside-down; an approach that often bears fruits - but other kinds of fruits:

\footnotetext{
${ }^{12}$ Latin for nothing toward the matter; extraneous.
} 

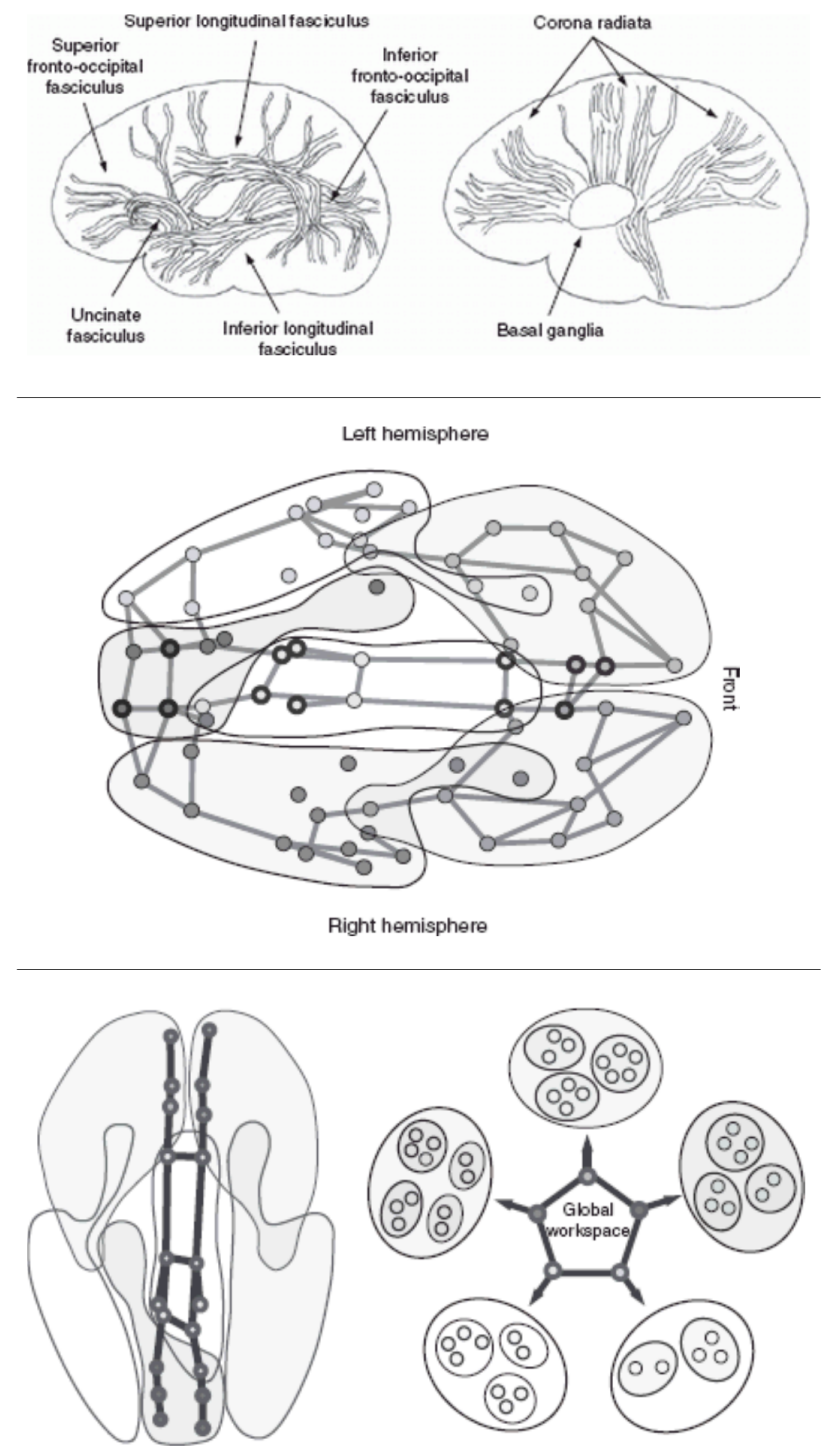

FIGURE 3.5: Global neural workspace in brain Top: White matter tracts. Left: Direct cortico-cortical pathways provide rich connectivity between the frontal, temporal, and occipital lobes are a likely substrate for a GW. Right: Thalamocortical pathways relay traffic to/from different parts of cortex, may also play a role in the putative workspace. Middle: Structural connectivity in the human brain. Each node corresponds to a cortical sub-region. Connector hubs are outlined in bold. Down: The brain's connective core as the possible locus of a neural GW. Left: Evidence suggests human cortex has a connective core running along its medial axis. Right: The connective core of a hierarchically modular network is topologically well placed to realize both broadcast (influence funnels into and fans out from the centre) and competition (it acts as a limited capacity bottleneck). So the connective core (left) is a candidate for the anatomical locus of a GW. Adapted from (Shanahan, 2010, figs 4.9-4.11). 
Many questions have found new answers once they were turned upside down. In medicine, for example, important discoveries have been made when, instead of asking "Why did this disease inflict this person now?" someone rather asked "Why doesn't this disease always inflict all people?" Bernard Baars ... tries to do something similar with the mind-body problem. Whereas most people often ask, "Why are there conscious experiences accompanying some brain processes?", he asks, "Why aren't there conscious experiences accompanying all brain processes?" (Elitzur, 1997, pp. 319-320)

Put differently, it seems that the GWT is so fixated on crafting (mechanistic) explanans that it overlooks explication of what consciousness is - and the whatquestions remain unanswered.

Elitzur (1997) then points out to Frank Jackson's Knowledge Argument (1982); the famous thought experiment about Mary, a vision neuroscientist, who has been trapped in a black and white room her whole life - hence has never seen any colorsbut knows every scientific fact about color red and the human visual system. The question raised by this thought experiment is whether Mary will gain any additional knowledge about color red if she exits the room and sees a red object. Many would agree that before leaving the room, she is missing something: the phenomenal experience or quale of color red ${ }^{13}$. The GWT does not address whether Mary's new knowledge differs from what she already had. Moreover, it virtually ignores $Q_{\text {phenomenal - what is unique about Mary's new experience, which many philosophers }}$ think increases her knowledge of color red?

\subsubsection{The missed desideratum: What happened to the Hard Problem?}

The other critique against the GWT is that, although it outlines the limits of what consciousness can or cannot be, it remains silent with regards to $Q_{\text {phenomenal }}$ - one of the most puzzling problems of consciousness. Although Baars could have cast aside the issue altogether by taking an eliminative stance towards it, like that of the MDM (cf. Section 3.2.3), he mostly prefers to remain a mugwump by respecting the privacy of the first-person experience. ${ }^{14}$ Baars, in a recent review of the GWT, writes:

The same point [in response to the Dennettian reductio ad absurdum ${ }^{15}$ objection against GWT's theater metaphor] may be made for other standard philosophical critiques of scientific studies of consciousness. Most scientists and many philosophers reject such "in principle" arguments against empirical work. As we have already noted, GWT is based on comparisons between brain events that support conscious experiences

\footnotetext{
${ }^{13}$ See (Nida-Rümelin, 2015) for a detailed discussion on this thought experiment and its implications.

${ }^{14}$ However, e.g., in (Baars et al., 2003), he has tried to apply the GWT to conscious experience.

${ }^{15}$ Also known as the appeal to extremes argument, whereby one tries to make a point by showing the (most) absurd conclusions and implications of her statement not being true.
} 
vs. those that do not, such as cortical vs. cerebellar activity. All of GWT is based on comparisons between clearly physical phenomena, though the conscious ones obviously are marked by phenomenological privacy from the perspective of the individual. Thus the standard critiques about qualia, the "Hard Problem," and so on, simply do not apply. (Baars, 2017, pp. 238-239)

One might wonder whether SGWM is immune to these objections. Albeit Shanahan makes use of inner language in the model, before painting his oeuvre, Shanahan (2010, Chapters 1 and 2) attempts to distance himself from the Hard Problem by utilizing Wittgenstein's approach of dissolving certain questions instead of solving them ${ }^{16}$. Hence, SGWM is susceptible to the two objections mentioned here; Shanahan primarily pencils an outline for a cohesive mechanistic account of the GWT yet falls short of solving these issues. For a concise overview of the latter issue and similar criticisms against SGWM, see (Aleksander, 2011).

Having in mind the issues raised in this section, one might wonder whether the GW approach toward consciousness is a (perhaps fundamentally) futile effort - or as said in Persian, an act of mourning upon an empty grave. Chapter 4 tries to prove otherwise by discussing the computational implementations of GWMs and Chapter 5 points out to implications of GWT for machine consciousness.

\footnotetext{
${ }^{16}$ For instance, in the first chapter, Shanahan writes the following and builds the second chapter on this basis:

But the opening chapter of the present book belongs to the relatively youthful tradition of trying, not to solve such problems, but to dissolve them. According to this way of thinking, which was inaugurated by Wittgenstein, a fog of metaphysical confusion is liable to obscure a proper view of mind. We cannot hope to find the desired scientific explanations amid this fog, forever wandering from one ism to another (dualism, materialism, cognitivism, ... ). Yet to emerge from the fog is to return to a kind of prelapsarian state, a state before philosophy, which is also a state after philosophy. (2010, p. 2)
} 



\section{Chapter 4}

\section{Computational Implementations of the Global Workspace Theory}

Better to roam the deserts than to frivolously dwell; For, if I do not attain my desire, I strive to the extent of my vigor.

— SA'ADī OF SHĪRĀZ (CA. 1210-1291)

The GWT has been fairly - if not greatly - successful in explaining aspects of consciousness. As discussed in Chapter 3, SGWM further connects explanations and predictions of the GWT to plausible neural realizations. Despite their limitations, GWMs are good candidates for implementations of "conscious" machines as they are in line with at least three strands of MC research, viz. MC1-3 (cf. Chapter 1, Section 1.3). The computational implementations of GWT (CGWMs), more specifically, IDA and LIDA, have been shown to exhibit behaviors attributed to conscious creatures (MC1), imitate cognitive processes (e.g., attention shift; MC2), and have cognitive architectures associated with consciousness (MC3). Moreover, in the light of Chapter 2, CGWMs also benefit the broader field of consciousness research since they can be used as "empirical" benchmarks for a set of mechanistic explanations of consciousness (see, e.g., Franklin et al., 2012, pp. 37-42).

The GWT is a functional framework hence benefits from the MR principle (cf. Section 2.3.1). This implies that the (C)GWMs are not limited to architectures similar to SGWM which explicitly involved a connective core as a substrate for the GW; a model can count as GWM if it emulates the main functionality of a GW (i.e., bottlenecking and broadcasting) to realize the GAH. ${ }^{1}$ The IDA and the

\footnotetext{
${ }^{1}$ This excerpt from (Franklin et al., 2012) emphasizes that (L)IDA benefits from MR - which can also be generalized to other CGWMs:

LIDA is [a] model of minds, not a model of brains. Our studies of empirical neuroscience [serve] as a part of the theoretical basis for our model. The recently initiated experimental arm of our work will test our model's ability to replicate the results of psychological experiments as well as its suitability for the design of autonomous agents that use human-like approaches to problem-solving. We have no intention of, nor will our work be capable of, directly testing our neuroscientific hypotheses. In other words,
} 
LIDA architectures realize such mechanism in a multi-agent framework. In this short chapter, the architectures of IDA and her heiress (i.e., LIDA) are reviewed.

\subsection{IDA}

IDA (Intelligent Distribution Agent; Franklin, Kelemen, \& McCauley, 1998) was the first attempt to computationally realize GWMs by incorporating a GW in an earlier intelligent autonomous agent framework (Franklin, 1997; Franklin \& Graesser, 1997). IDA was developed for the US Navy to assign sailors to new billets based on their performance in their previous duty tours, taking into account sailors' needs and desires by asking their opinion in natural language. IDA consists of modules that are loosely named after the cognitive functions they are meant to serve, such as perception, emotion, deliberation, metacognition, and "consciousness." Figure 4.1 (adapted from Franklin, 2003, fig. 2) shows the IDA architecture. Each module is realized by codelets, which are special-purpose, semi-autonomous mini-agents implemented as small pieces of code running on separate threads. These codelets are idle unless their required conditions are met ${ }^{2}$. The codelets resemble the processes of coalitions in the GWT since they can "unconsciously" run in parallel and share their states or informational contents with others.

According to Franklin (2003), these modules only partially resemble their neurocognitive counterparts. For instance, the "perception" module, which is responsible for accessing - and making sense of - the (textual) inputs (i.e., the database and the emails she receives from sailors) is preconscious. Hence IDA's "perception" differs materially from the human perception studied in psychology wherein conscious awareness is an integral element. Additionally, human comprehension of natural language is mostly conscious (and engages the GW) while IDA "understands" the incoming emails by simple surface-level natural language processing techniques - which is substantially different from that of humans. ${ }^{3}$ Other modules are very simple functional implementations of (supposedly) real cognitive processes. For instance, IDA has an "emotion" module, enabling her to "experience' such emotions as guilt at not getting a sailor's orders out on time, frustration at not understanding a message, and anxiety at not being able to convince a sailor to accept a suitable job." (Franklin, 2003, p. 55) Despite its simplicity, the emotion module is a key component of IDA; it influences action selection (by affecting the drives) as well as attention (via bringing certain content to "consciousness") and the strength of consolidation of events in her long-term memory.

IDA's GW is mostly reduced to episodic memory and does not fully echo that of Baars' model (cf. Section 3.2.2, footnote 4). On the other hand, her "consciousness" apparatus consists of "a coalition manager, a spotlight controller, a broadcast manager,

we neither require nor expect that LIDA's internal dynamics correspond to those of brains, only that its functions match the cognitive functions of minds. (p. 33)

\footnotetext{
${ }^{2}$ The conditions can be being triggered by other codelets (e.g., receiving their information) or states or levels of activity of other modules.

${ }^{3}$ See Franklin's discussion (2003, pp. 53-54) on attributing this term to the perception module.
} 


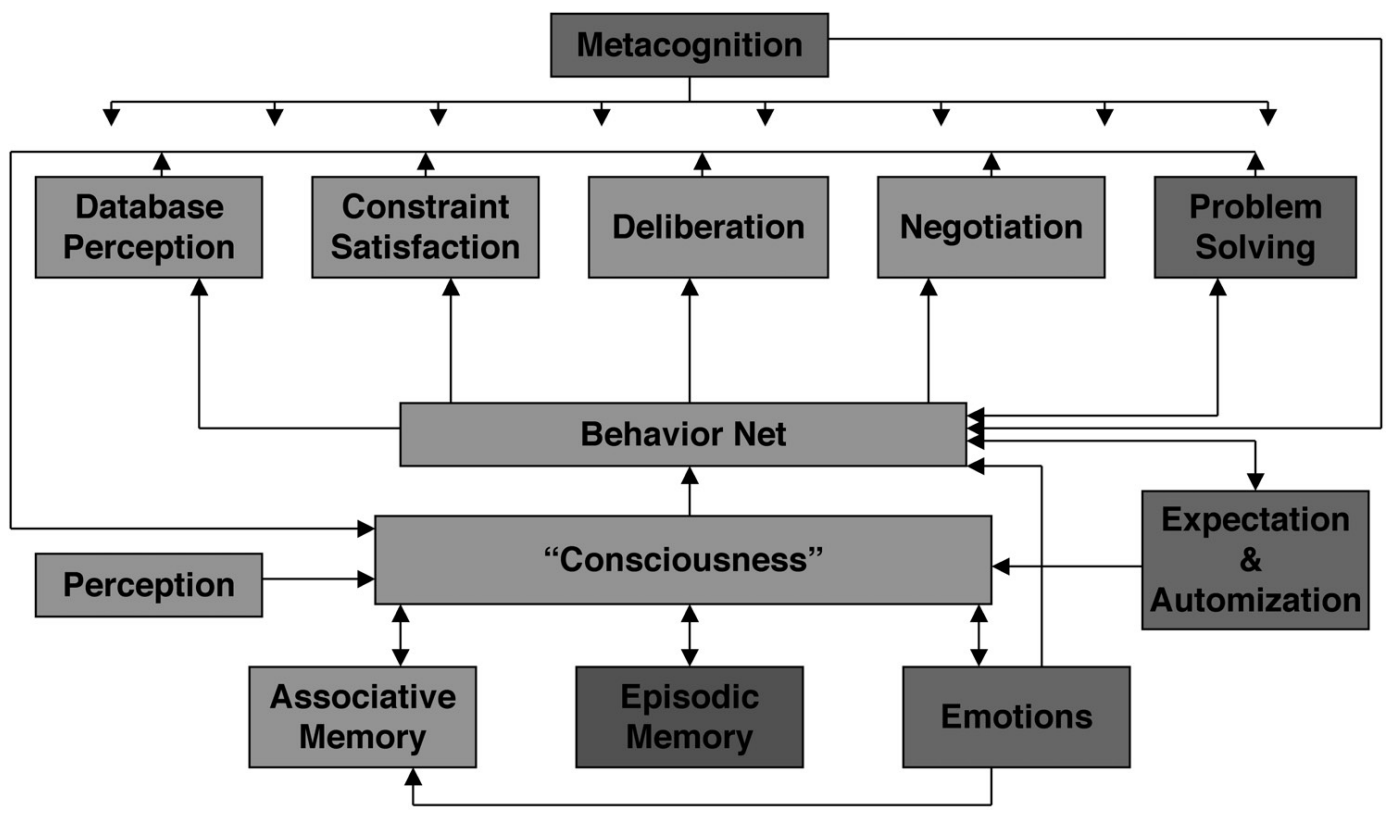

Figure 4.1: The IDA architecture It consists of specialized modules. The box labeled as "consciousness" carries out the bottlenecking-broadcasting function of the GW, and is connected to perception (the natural language input and database), emotion, and memory modules. A behavior net mediates between the GW and other cognitive modules. Adapted from (Franklin, 2003, fig. 2).

and a collection of attention codelets whose job is to bring appropriate contents to "consciousness." (Franklin, 2003, p. 55) Hence, IDA's consciousness module is merely a realization of the bottleneck-broadcast mechanism (Figure 3.1), but the "stage" of the theater (Figure 3.3) falls outside of her GW. Yet, as emphasized earlier in this section, this, per se, does not blackball IDA from the family of (C)GWMs.

The IDA architecture, with all its technical complexities, is a relatively simplistic implementation of the GWT and is far off the so-called biologically-inspired cognitive architectures (BICAs). Intelligent organisms, most notably, have three properties: they are situated in their environment, they are embodied in a biological body, and they dynamically learn from their experiences. ${ }^{4}$ IDA lacks these properties, and it cannot be remedied by adding (more complex) modules within her current architecture. Learning is acquired by constant exposure of the agent to the environment (i.e., other agents and objects, and the consequences of her own actions in the environment), and via the plasticity of the agent to learn from her experiences. Neither of these can be attained in IDA - and this is the departure point of LIDA.

\footnotetext{
${ }^{4}$ See (Pedersen, 2016) for a detailed discussion about SED (situated, embodied, and dynamic) approach in AI.
} 


\subsection{LIDA}

The Learning IDA (LIDA) architecture is a situated cognitive model that adds three modes of learning to IDA, namely, perceptual, episodic, and procedural learning (Franklin \& Patterson, 2006). It posits that cognitive life of intelligent organisms consists of consecutive cognitive cycles of sampling the environment (i.e., sensing) and responding accordingly (i.e., acting). Each cycle - that can be thought of as an atomic cognitive "moment"-is comprised of three phases, namely, understanding, attending, and acting. Franklin and colleagues write:

Just as atoms have inner structure, the LIDA model hypothesizes a rich inner structure for its cognitive cycles (Baars \& Franklin, 2003; Franklin, Baars, Ramamurthy, \& Ventura, 2005). During each cognitive cycle[,] the LIDA agent first makes sense of its current situation as best as it can by updating its representation of both external and internal features of its world. By a competitive process ... it then decides what portion of the represented situation is most salient, that is, in the most in need of attention. This portion is broadcast, making it the current contents of consciousness, and enabling the agent to choose an appropriate action and execute it. Though GWT speaks to phenomenal consciousness, and thus to the "hard problem" of consciousness (Chalmers, 1996), the LIDA model follows Shanahan (2010) in taking the "postreflective inner view" and doing "... without the habit of metaphysical thinking". More specifically, consciousness in the LIDA model refers to functional consciousness in which no assumption is made of the conscious broadcast being phenomenally conscious (Franklin, 2003). (Franklin et al., 2012, p. 35)

The LIDA cognitive cycle (adapted from Franklin et al., 2016, fig. 2) is shown in Figure 4.2. This cycle allows LIDA to learn dynamically. Moreover, the LIDA architecture can host situated cognition thanks to its additional features (see Franklin et al., 2016, p. 108 for details). The LIDA architecture is highly convoluted, hence, and in the interest of brevity, it will not be discussed anymore. The keen reader may consult Franklin et al. (2016) for a comprehensive tutorial of LIDA.

This chapter ends here after introducing IDA and LIDA as two computational implementations of the GWT. However, any model of MC has to address the philosophical problems raised against artificial consciousness. In the next chapter, one of such problems, namely, the Frame Problem, is discussed and applied to the GWT. 


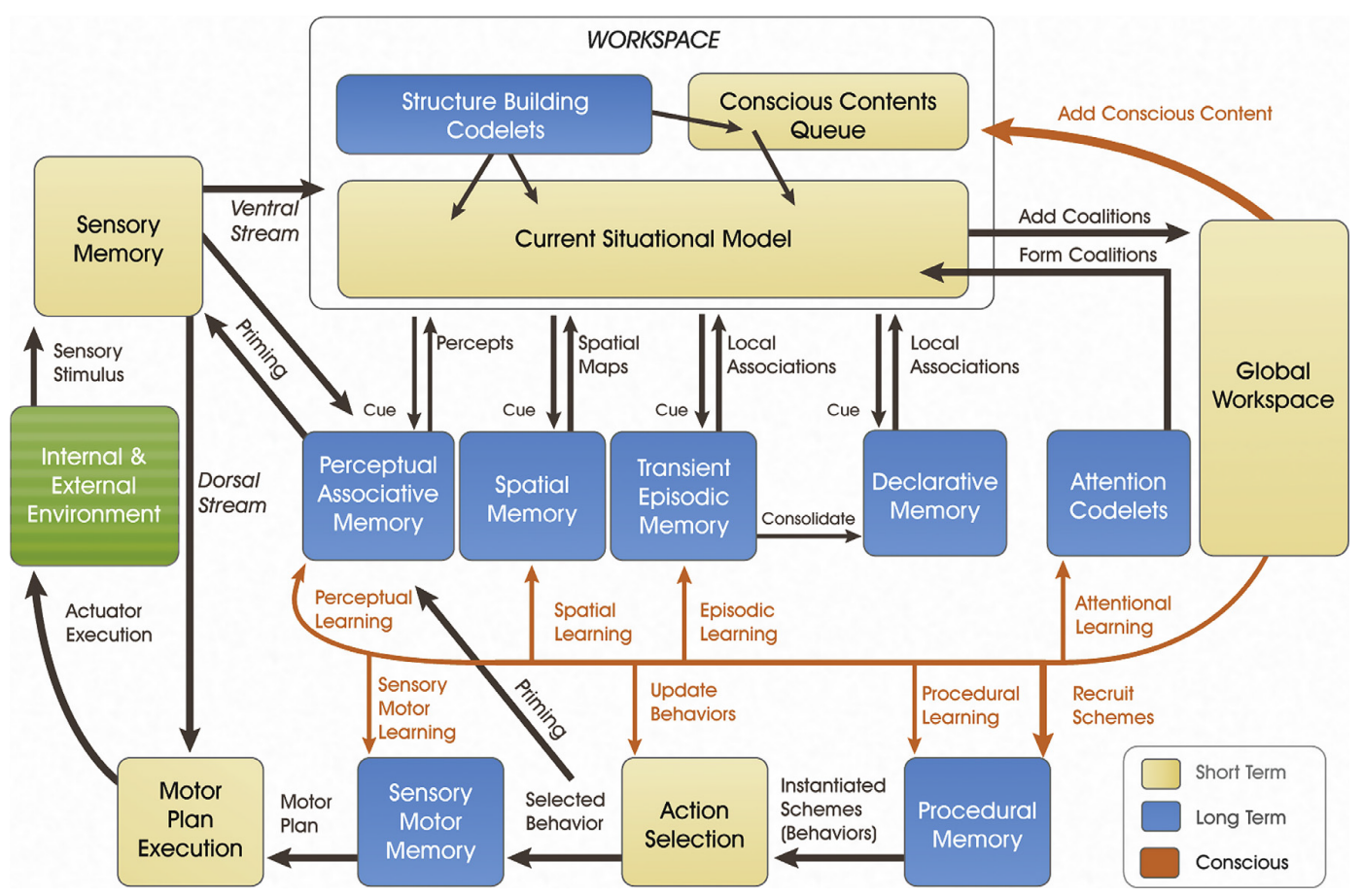

Figure 4.2: The LIDA cognitive cycle Adapted from (Franklin et al., 2016, fig. 2 ). Find the description of the diagram in the source. 



\section{Chapter 5}

\section{Can Machines Ever Become Conscious?}

Happy it is if the touchstone of experience comes into use; So that ignominious becometh every one in whom is alloy.

— Ḥִ̂̄FeZ of ShĪRĀZ (CA. 1315-1390)

The history of AI is a story of shooting for the stars. Originally, the goal of AI was "replicating" machines that can resemble human intelligence as much as possiblehoping that they will someday be indistinguishable from that of their creators. The resurgence of consciousness research in the recent decades has shed light on what that goal really means; or, what would - or would not - count as resemblance to human intelligence - and where to set the bar for our success in developing intelligent machinery. The philosophers, cognitive scientists, and computer scientists have teamed up into two groups: those who cherish that AI would attain most (if not all) aspects of human consciousness, most importantly, in line with MC4, phenomenal consciousness; and those who believe that such goal, at least for the current computing machinery (i.e., the Turing machines; see De Mol, 2018), is not feasible - if not impossible. The former idea is known as Strong AI and the latter as Weak AI.

The proponents of Weak AI doubt the aims of Strong AI either based on practical limitations of AI (most importantly, the fact that even the most prominent implementations of AI can only host quite narrow, domain-specific tasks), or based on philosophical arguments that set hard limits on capabilities of AI - and its semblance to human consciousness. The philosophical arguments are usually of the form of what is known in mathematics and logic as reductio ad impossibile ${ }^{1}$; they show that Strong AI entails consequences that are logically impossible or (highly) counterintuitive.

The first group of such philosophical arguments are about capabilities (capability objections; COs); they involve addressing things that humans can do but machines

\footnotetext{
${ }^{1}$ Also known as indirect proof or proof by contradiction, where, in order to prove a proposition $p$, it is shown that $p$ being false entails contradiction with other propositions.
} 
cannot, hence concluding that intelligence is not computational. The second group of such arguments concern properties of consciousness (property objections; POs); they try to show that certain (intuitive) aspects or properties of consciousness cannot be realized computationally as their entailments would contradict those properties. Most important of these properties are $C_{\text {creature }}^{\text {what-it-is-like }}, C_{\text {statie }}^{\text {quative }}, C_{\text {creature }}^{\text {phenol }}$, free will, personhood, and identity.

POs root in one's assumptions about such properties, e.g., the nature of free will, identity, or qualia. Consequently, the counter-arguments against POs usually involve questioning those premises (e.g., via Dennettian qualia and self eliminativism; see Section 3.2.3 on the MDM). The COs, on the other hand, do not depend on such contentious premises as they only rely on attributing specific capabilities to humans. Hence, the COs are more general and can readily be presented formally. Moreover, some COs are also discussed outside the literature of consciousness research as well. The counter-arguments against these COs often doubt the attribution of those capabilities to humans. In Section 5.1 one of the COs (namely, the frame problem) is introduced, and the challenges it imposes on cognitive theories of mind (and consequently, Strong AI) are discussed. Afterward, Section 5.2 discusses how the GWT deals with the frame problem and how it benefits cognitive theories and Strong AI. The next chapter will summarize and conclude the thesis.

\subsection{The Frame Problem: A Touchstone for (Models of) Consciousness}

The Frame Problem (FP) is primarily an epistemological problem which first sprouted in logic-based AI as a practical issue. It was later welcomed in logic and philosophy, and its branches spread to other fields such as cognitive science - most importantly, the computational theory of mind (CTM), which posits that cognitive processes are computational wherein "mental states comprise sets of propositional attitudes and mental processes are forms of inference over the propositions in question." (Shanahan, 2016, sec. 3; see also Rescorla, 2017) ${ }^{2}$

More specifically, the FP concerns the consequences of actions in reasoning based on first-order logic. To present the FP, suppose an agent $A$ has a set of logic formulae in her knowledge base $K$ that formally describes her world $(W)$, i.e., her environment (its elements and the relations between the elements), her place in the environment, and her relation to elements of that environment. If the agent is autonomous, she should be able to make decisions on her own by evaluating the consequences of her action in $W$. In the logic-based AI, this evaluation is logical reasoning based on the axioms and formulae she has in $K$. Shanahan and Baars (2005) explain where the FP starts:

How is it possible to write a collection of axioms in mathematical logic that captures the effects of actions, without being obliged to include an

\footnotetext{
${ }^{2}$ Note that the CTM is not an integral part of the ETLM nor GWMs per se-although they may be (remarkably) inclined towards the CTM.
} 
overwhelming number of axioms that describe the trivial non-effects of those actions? In everyday discourse, we can describe the effect of, say, painting an object simply by detailing how its color changes. There is no need to state explicitly that the object's shape remains the same, that the neighbor's cat remains asleep, that the Sun continues to shine, and so on. This is all taken for granted by common sense.

In mathematical logic, by contrast, nothing is taken for granted. Not only is it necessary to make explicit the changes an action brings about, it is also necessary to make explicit the things that do not change, and for most actions this will be a great deal. This was particularly troubling for AI researchers in the 1970s and 1980s who wanted to write programs that worked by carrying out inference with logical formulae. The burden of having to supply a set of explicit formulae describing every non-effect of every action seemed overwhelming, both representationally and computationally. (p. 158, with minor spelling edits.)

An intuitive approach to rule out the non-effects is assuming the common-sense law of inertia; actions do not change properties of the situation unless there is evidence against it. However, applying this law is not as trivial as it seems; adding new formulae to $K$ always increases the number of conclusions that can be deduced from it. This property of classical logic is known as monotonicity (Shanahan, 2016, sec. 2 ). Due to monotonicity, adding the law of inertia to $K$ makes the problem even worse; before adding the law of inertia, $A$ had to go over all possible combination of axioms in $K$ before deciding which action to carry out, but now she has to "think" when to apply the law of inertia in her reasoning. Hence, the FP is framing the relevant effect axioms out of the myriad non-effect axioms.

Efforts have been made to solve this problem by developing non-monotonic reasoning formalisms - which has not been straightforward. In computer engineering, however, this problem is practically "solved" in the typical applications - e.g., by utilizing appropriate heuristics in reasoning - but the epistemological problem remains (Shanahan, 2016). Consequently, the FP can be used as a CO against Strong AI; the absolute majority - if not all - of humans do not fall into the "infinite regress" of $\mathrm{FP}$ - but machines do. However, a counter-argument against this $\mathrm{CO}$ is that it is built upon two premises: (1) humans are not prone to the infinite regress, and (2) humans do not make mistakes in their reasoning. The second premise is not always true - "to err is human." Hence, if we relax the latter premise for machines too, i.e., by letting them make mistakes from time to time, then the epistemological $\mathrm{FP}$ reduces to a practical $\mathrm{FP}^{3}$ - for which adequate solutions already exist.

\footnotetext{
${ }^{3}$ Shanahan explains this point:

One way to mitigate the threat of infinite regress is by appeal to the fact that, while humans are more clever than today's robots, they still make mistakes (McDermott 1987). People often fail to foresee every consequence of their actions even though they lack none of the information required to derive those consequences, as any novice chess player can testify. Fodor asserts that "the frame problem goes very deep; it goes
} 
The above counter-argument only benefits logic-based AI against the FP objection under a very specific condition: that the framing is over purely logical reasoning, solely over logical axioms. However, many cognitive processes, like analogical reasoning ${ }^{4}$, are intrinsically informationally unencapsulated, i.e., they draw on information from arbitrary domains of knowledge or expertise (Shanahan, 2016). Such processes are thought to be computationally infeasible. Shanahan and Baars (2005) cite Fodor and Carruthers on this matter:

The totality of one's epistemic commitments is vastly too large a space to have to search if all one's trying to do is figure out whether, since there are clouds, it would be wise to carry an umbrella. Indeed, the totality of one's epistemic commitments is vastly too large a space to have to search whatever it is that one is trying to figure out. (Fodor, 2000, p. 31)

The computational processes that realize human cognition will need to be tractable ones, of course; for they need to operate in real-time with limited computational resources ... And any processor that had to access the full set of the agent's background beliefs (or even a significant subset thereof) would be faced with an unmanageable combinatorial explosion. (Carruthers, 2003, p. 505, with minor spelling edits.)

Fodor concludes that cognitive processes are not computational - at least in general. More specifically, he distinguishes between informationally encapsulated and unencapsulated processes. According to Shanahan and Baars,

Famously, such considerations lead Fodor to propose a distinction between the mind's peripheral processes, which are supposedly informationally encapsulated and therefore computationally feasible, and its central processes, which are informationally unencapsulated and therefore computationally infeasible. The mind's peripheral processes - which do only "moderately" interesting things, like parsing and early vision-can be understood as a system of modules, and are amenable to study by cognitive scientists. On the other hand, the mind's central processeswhich do all the really interesting and important things like belief revision

as deep as the analysis of rationality" (Fodor 1987). But the analysis of rationality can accommodate the boundedness of the computational resources available to derive relevant conclusions (Simon 1957; Russell \& Wefald 1991; Sperber \& Wilson 1996) Because it sometimes jumps to premature conclusions, bounded rationality is logically flawed, but no more so than human thinking. However, as Fodor points out, appealing to human limitations to justify the imposition of a heuristic boundary on the kind of information available to an inferential process does not in itself solve the epistemological frame problem (Fodor 2000, Ch.2; Fodor 2008, Ch.4; see also Chow 2013). This is because it neglects the issue of how the heuristic boundary is to be drawn, which is to say it fails to address the original question of how to specify what is and isn't relevant to the inferential process. (2016, sec. 3)

${ }^{4}$ e.g., finding an analogy between a Rorschach inkblot pattern and an object, animal, or concept. See (Shanahan \& Baars, 2005, pp. 167-168). 
and analogical reasoning - because they are computationally infeasible are altogether beyond understanding for contemporary cognitive science. (2005, p. 161)

If Fodor and his sympathizers are right-i.e., that the FP of informationally unencapsulated processes is computationally infeasible hence seriously imperil the CTM - then Strong AI would be at risk as well; Strong AI and the CTM share the same crux: that cognitive processes are, in essence, computational. ${ }^{5}$

Shanahan and Baars (2005) argue that this concern is valid only if the CTM is to be realized within a naïve serial architecture (as Fodor and others had conceptualized). Hence, they argue, that a GW architecture can rescue the CTM from the FP.

\subsection{The Frame Problem in a GW Architecture}

Shanahan and Baars (2005, pp. 167-173) go into great details to discuss analogical reasoning and its relevance to the CTM and the FP. ${ }^{6}$ They compare various computational models of analogical reasoning suggested in the literature with an emphasis on the computational complexity of such models. These models share the same linchpin of a centralized serial Fodorian model (see below) and they differ, e.g., in the heuristics or algorithmic structures they utilize to make the model more efficient. Shanahan and Baars conclude that the serial models have a time complexity of the order $O\left(n^{2} m^{2}\right)$, where $n$ is the total number of candidate base representations (e.g., objects that resemble the inkblot) and $m$ is the number of propositions in the largest base or target representation. Let us take a look at the serial architecture before comparing it to that of the GW.

The Fodorian serialized architecture consists of peripheral modules that respond to a central unit when they are asked about the relevance of their content to the problem at hand. See Figure 5.1 (adapted from Shanahan \& Baars, 2005, fig. 2). Although the peripheral modules are "placed" in parallel, they are idle until the central module calls them (Shanahan \& Baars, 2005, pp. 167-168) and this inquiry is carried out

\footnotetext{
${ }^{5}$ However, note that "[the CTM] is stronger than the claim that the input-output function of every human brain is equivalent to some Turing machine. As Fodor puts it, CTM entails that "the mind is interestingly like a Turing machine' (Fodor, 2000, p. 30).” (Shanahan \& Baars, 2005, p. 160) Consequently, one might argue that failure of the CTM in addressing the FP only entails the failure of Strong symbolic AI-while the non-symbolic, connectionist paradigm can still prosper in attaining Strong AI.

${ }^{6}$ Shanahan and Baars write:

The Rorschach inkblot example is especially pertinent, since it is closely related to analogical reasoning. As Fodor emphasizes, reasoning by analogy is the epitome of an informationally unencapsulated cognitive process because it "depends precisely upon the transfer of information among cognitive domains previously assumed to be irrelevant" (Fodor, 1983, p. 105). Moreover, analogical reasoning is arguably central to human cognitive prowess (Gentner, 2003). So we should be able to offer a prima facie case that the global workspace architecture relieves the computational burden allegedly brought on by the informational unencapsulation of analogical reasoning. (2005, p. 168, with minor spelling edit)
} 
Peripheral Processes (Modules)

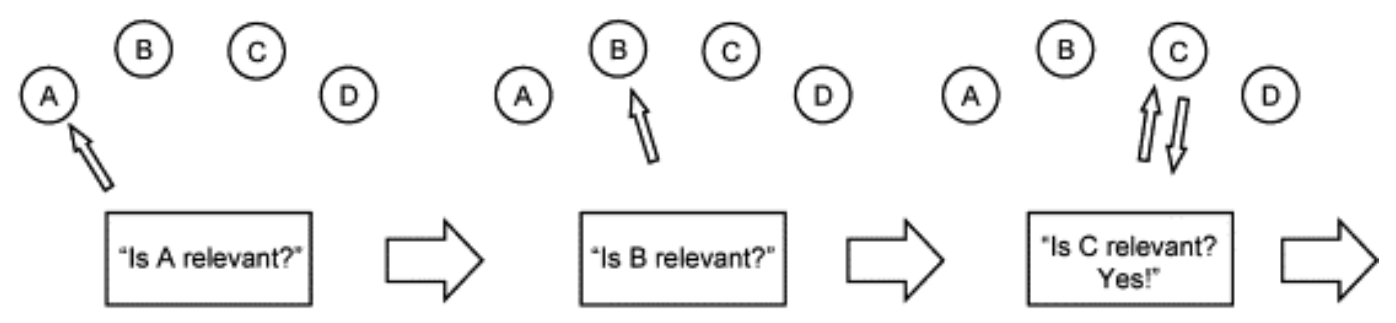

Central Processes

FiguRE 5.1: A naïve model of serialized information flow The peripheral processors are informationally encapsulated, hence computationally feasible. The central processor "asks" the peripheral processors, one-at-a-time, whether they are relevant to the reasoning. The centralized process is informationally unencapsulated, hence not computationally feasible. Adapted from (Shanahan \& Baars, 2005, fig. 2).

sequentially. This architecture "outsources" informationally encapsulated processes to the peripheral modules, but the informationally unencapsulated residual of the processes are left to the central module. Consequently, the FP continues to reside at the heart of the central module, hence making the architecture computationally infeasible.

The GW architecture, on the other hand, is different from the Fodorian architecture in at least two ways: (1) in the GWM, the modules are autonomous and running in parallel, and (2) the inquiry initiated by the central module (i.e., the $\mathrm{GW}$ ) is broadcast to all of them at the same time. The relevance of the processes of the modules to the question at hand is then determined locally by the modules on their own (Figure 5.1; adapted from Shanahan \& Baars, 2005, fig. 2). This rules out the main FP which rooted in the serialized comparison of processes. Yet, further reasoning based on the relevant processes has to happen serially - which is in accord with the bottleneck property of the GW. Shanahan and Baars give an estimate of $O(\log m)$ for the time complexity of the GW parallel architecture where $m$ is defined similarly to the serialized architecture.

Although still computationally demanding, the GW architecture is considerably more efficient than the Fodorian implementation. More importantly, the GW reduces the "mystery" of realization of the FP in the CTM to a scientific "problem" of understanding how the remainder of the processes is (more efficiently) implemented in the brain. The GW solution to the FP in the CTM shields Strong symbolic AI from the capability objection based on the FP (cf. footnote 5 earlier in this chapter). This point concludes the thesis, and the next chapter will summarize what has been discussed so far. 
Parallel Unconscious Specialists

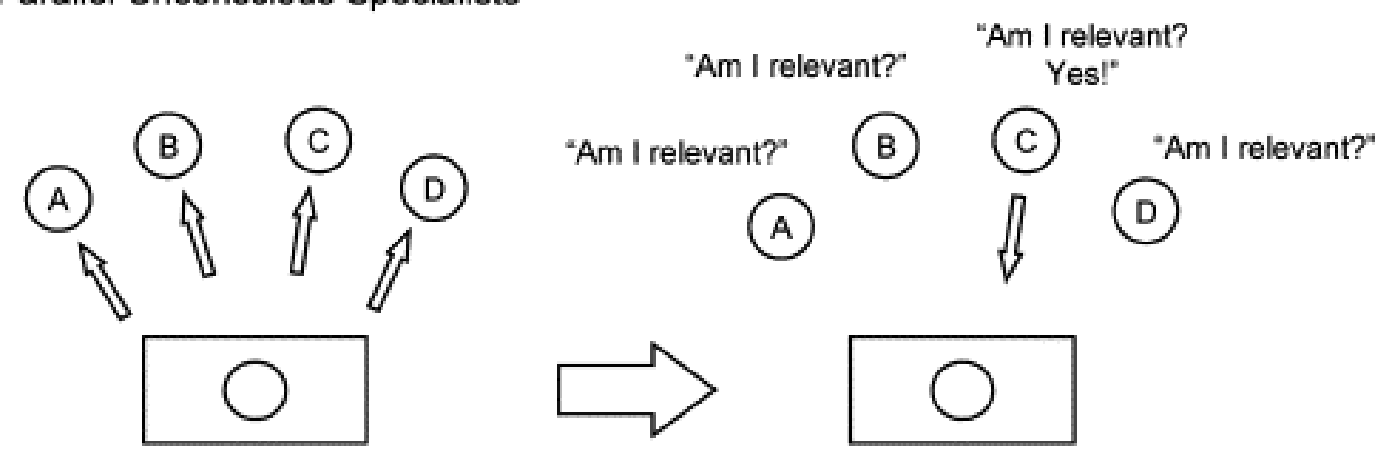

\section{Global Workspace}

FIGURE 5.2: The global workspace model of information flow The GW broadcasts the problem to the autonomous processes running in parallel. The process relevant to the problem at hand responds to the inquiry. Adapted from (Shanahan \& Baars, 2005, fig. 3). 



\section{Chapter 6}

\section{Conclusion}

In the time of separation from thee, what I endured, alas Is impossible that, in one letter, writing thereof I should make.

This thesis had two main goals: introducing a promising neurocognitive theory of human consciousness (viz., the global workspace theory; GWT), and showing how it can benefit the study of consciousness in machines. To reach these goals, Chapter 1 showed a bird's-eye view of the terrains of philosophy of mind - so the reader gets acquainted with the land upon which they will set foot - and briefly introduced what it means to study consciousness in machines. Chapter 2 provided the reader with a powerful toolset of mechanistic explanations for their journey. In this chapter, the tri-level approach of studying human mind was introduced; a versatile framework that helps systematically study aspects of consciousness by bringing various strands of consciousness research together and easing their dialogue under that framework.

Chapter 3 gave an introduction to the neurocognitive theories of consciousness. It reviewed the efforts in bringing neuroscience and philosophy of mind closer to each other. In the same chapter, the GWT was discussed in details. The GWT, together with the tri-level mechanistic approach of Chapter 2, can connect many dots between neuroscience, cognitive science, and artificial intelligence. In Chapter 4, two computational models of the GWT were introduced as proofs of concept for computational implementation of certain aspects of consciousness.

Finally, Chapter 5 discussed a deep philosophical problem limiting artificial intelligence, viz., the Frame Problem (FP). It showed that the GWT breaks the spell of the FP and opens horizons for the ambitions of Strong AI. The pinnacle of this dissertation was towards the end of Chapter 5 where the power of the GWT against the FP and its important implications for machine consciousness (MC) were revealed.

This thesis was a small step in introducing the GWT and mechanistic explanations to the study of MC. Nevertheless, the author hopes that it will encourage further research into the crossroad of the GWT and MC. 



\section{References}

Adriaans, P. (2019). Information. In E. N. Zalta (Ed.), The stanford encyclopedia of philosophy (Spring 2019). Metaphysics Research Lab, Stanford University. Retrieved from https://plato.stanford.edu/archives/spr2019/entries/ information/

Aleksander, I. (2011). Workspace theories are alive and well. International Journal of Machine Consciousness, 03(2), 309-312. http://doi.org/10.1142/ S1793843011000704

Andrianantoandro, E., Basu, S., Karig, D. K., \& Weiss, R. (2006). Synthetic biology: New engineering rules for an emerging discipline. Molecular Systems Biology, 2(1), 2006.0028. http://doi.org/10.1038/msb4100073

Artificial intelligence (AI) coined at Dartmouth. Dartmouth College. (n.d.). Retrieved August 2, 2019, from http://archive.is/yVq1J

Audi, P. (2015). Explanation and explication. In C. Daly (Ed.), The palgrave handbook of philosophical methods (pp. 208-230). London: Palgrave Macmillan UK. http://doi.org/10.1057/9781137344557_9

Baars, B. J. (1988). A cognitive theory of consciousness. Cambridge University Press.

Baars, B. J. (1997a). In the theater of consciousness: The workspace of the mind. New York: Oxford University Press.

Baars, B. J. (1997b). In the theatre of consciousness: Global workspace theory, a rigorous scientific theory of consciousness. Journal of Consciousness Studies, 4(4), 292-309.

Baars, B. J. (2002). The conscious access hypothesis: Origins and recent evidence. Trends in Cognitive Sciences, 6(1), 47-52. http://doi.org/10.1016/ S1364-6613(00)01819-2

Baars, B. J. (2017). The global workspace theory of consciousness: Predictions and results. In S. Schneider \& M. Velmans (Eds.), The blackwell companion to consciousness (pp. 229-242). Chichester, UK: John Wiley \& Sons, Ltd. http://doi.org/10.1002/9781119132363.ch16 
Baars, B. J., \& Franklin, S. (2003). How conscious experience and working memory interact. Trends in Cognitive Sciences, 7(4), 166-172. http://doi.org/10. 1016/S1364-6613(03)00056-1

Baars, B. J., Ramsøy, T. Z., \& Laureys, S. (2003). Brain, conscious experience and the observing self. Trends in Neurosciences, 26(12), 671-675. http://doi.org/ $10.1016 / j$.tins. 2003.09.015

Baker, A. (2016). Simplicity. In E. N. Zalta (Ed.), The stanford encyclopedia of philosophy (Winter 2016). Metaphysics Research Lab, Stanford University. Retrieved from https://plato.stanford.edu/archives/win2016/entries/ simplicity/

Bassett, D. S., \& Bullmore, E. (2006). Small-world brain networks. The Neuroscientist, 12(6), 512-523. http://doi.org/10.1177/1073858406293182

Baxendale, M. (2016). The layer cake model of the world and non-reductive physicalism. Kriterion - Journal of Philosophy, 30(1), 39-60.

Beckermann, A. (1992). Supervenience, emergence, and reduction. In A. Beckermann, H. Flohr, \& J. Kim (Eds.), Emergence or reduction?: Prospects for nonreductive physicalism (pp. 94-118). De Gruyter.

Bickle, J. (2019). Multiple realizability. In E. N. Zalta (Ed.), The stanford encyclopedia of philosophy (Spring 2019). Metaphysics Research Lab, Stanford University. Retrieved from https://plato.stanford.edu/archives/spr2019/ entries/multiple-realizability/

Block, N. (1992). Begging the question against phenomenal consciousness. Behavioral and Brain Sciences, 15(2), 205-206. http://doi.org/10.1017/s0140525x00068266

Bringsjord, S., \& Govindarajulu, N. S. (2018). Artificial intelligence. In E. N. Zalta (Ed.), The stanford encyclopedia of philosophy (Fall 2018). Metaphysics Research Lab, Stanford University. Retrieved from https://plato.stanford. edu/archives/fall2018/entries/artificial-intelligence/

Brysbaert, M., Stevens, M., Mandera, P., \& Keuleers, E. (2016). How many words do we know? Practical estimates of vocabulary size dependent on word definition, the degree of language input and the participant's age. Frontiers in Psychology, 7. http://doi.org/10.3389/fpsyg.2016.01116

Carruthers, P. (2003). On fodor's problem. Mind \& Language, 18(5), 502-523. http://doi.org/10.1111/1468-0017.00240

Carruthers, P. (2016). Higher-order theories of consciousness. In E. N. Zalta (Ed.), The stanford encyclopedia of philosophy (Fall 2016). Metaphysics Research Lab, Stanford University. Retrieved from https://plato.stanford.edu/archives/ fall2016/entries/consciousness-higher/ 
Chalmers, D. J. (1996). The conscious mind: In search of a fundamental theory. Oxford University Press.

Chalmers, D. J. (2010). The character of consciousness. Oxford University Press. http://doi.org/10.1093/acprof:oso/9780195311105.001.0001

Churchland, P. S. (1989). Neurophilosophy: Toward a unified science of the mindbrain. MIT Press.

Claeskens, G., \& Hjort, N. L. (2008). Model selection and model averaging. Cambridge ; New York: Cambridge University Press.

Combs, A. (1997). Commentary on bernard baars' "in the theatre of consciousness". Journal of Consciousness Studies, 4(4), 314.

Cooney, J. W., \& Gazzaniga, M. S. (2003). Neurological disorders and the structure of human consciousness. Trends in Cognitive Sciences, 7(4), 161-165. http: //doi.org/10.1016/S1364-6613(03)00058-5

Craver, C. F. (2001). Role functions, mechanisms, and hierarchy. Philosophy of Science, 68(1), 53-74. http://doi.org/10.1086/392866

Craver, C. F. (2007). Explaining the brain: Mechanisms and the mosaic unity of neuroscience. Oxford : New York : Oxford University Press: Clarendon Press.

Craver, C. F., \& Tabery, J. (2019). Mechanisms in science. In E. N. Zalta (Ed.), The stanford encyclopedia of philosophy (Summer 2019). Metaphysics Research Lab, Stanford University. Retrieved from https://plato.stanford.edu/archives/ sum2019/entries/science-mechanisms/

Dehaene, S., \& Changeux, J.-P. (2011). Experimental and theoretical approaches to conscious processing. Neuron, 70(2), 200-227. http://doi.org/10.1016/j . neuron.2011.03.018

Dehaene, S., Kerszberg, M., \& Changeux, J.-P. (2001). A neuronal model of a global workspace in effortful cognitive tasks. Annals of the New York Academy of Sciences, 929(1), 152-165. http://doi.org/10.1111/j.1749-6632.2001.tb05714.x

Dehaene, S., Sergent, C., \& Changeux, J.-P. (2003). A neuronal network model linking subjective reports and objective physiological data during conscious perception. Proceedings of the National Academy of Sciences, 100(14), 8520-8525. http: //doi.org/10.1073/pnas.1332574100

De Mol, L. (2018). Turing machines. In E. N. Zalta (Ed.), The stanford encyclopedia of philosophy (Winter 2018). Metaphysics Research Lab, Stanford University. Retrieved from https://plato.stanford.edu/archives/win2018/ entriesuring-machine/

Dennett, D. C. (1992). The self as a center of narrative gravity. In F. Kessel, P. Cole, \& D. Johnson (Eds.), Self and consciousness: Multiple perspectives (pp. 275-288). 
Erlbaum, Hillsdale NJ.

Dennett, D. C. (1993). Consciousness explained. Penguin UK.

Dennett, D. C. (2001). Are we explaining consciousness yet? Cognition, 79(1), 221-237. http://doi.org/10.1016/S0010-0277(00)00130-X

Dennett, D. C., \& Kinsbourne, M. (1992). Time and the observer: The where and when of consciousness in the brain. Behavioral and Brain Sciences, 15(2), 183-201. http://doi.org/10.1017/S0140525X00068229

Elitzur, A. C. (1997). Why don't we know what mary knows? Baars' reversing the problem of qualia. Journal of Consciousness Studies, 4(4), 319.

Fahimi Hnazaee, M., Khachatryan, E., \& Van Hulle, M. M. (2018). Semantic features reveal different networks during word processing: An EEG source localization study. Frontiers in Human Neuroscience, 12. http://doi.org/10.3389/fnhum. 2018.00503

Fodor, J. A. (2000). The mind doesn't work that way: The scope and limits of computational psychology. Cambridge, Mass: MIT Press.

Franklin, S. (1997). Autonomous agents as embodied ai. Cybernetics and Systems, 28(6), 499-520. http://doi.org/10.1080/019697297126029

Franklin, S. (2003). IDA: A conscious artifact? Journal of Consciousness Studies, 10(4), 47-66. Retrieved from www. ingentaconnect.com/content/imp/jcs/ 2003/00000010/f0020004/1346\#Refs

Franklin, S. (2011). Global workspace theory, shanahan, and lida. International Journal of Machine Consciousness, 03(2), 327-337. http://doi.org/10.1142/ S1793843011000728

Franklin, S., Baars, B. J., Ramamurthy, U., \& Ventura, M. (2005). The role of consciousness in memory. Brains, Minds and Media, 1(1), 1-38.

Franklin, S., \& Graesser, A. (1997). Is it an agent, or just a program?: A taxonomy for autonomous agents. In J. P. Müller, M. J. Wooldridge, \& N. R. Jennings (Eds.), Intelligent agents III agent theories, architectures, and languages (pp. 21-35). Springer Berlin Heidelberg.

Franklin, S., Kelemen, A., \& McCauley, L. (1998). IDA: A cognitive agent architecture. In SMC'98 conference proceedings. 1998 IEEE international conference on systems, man, and cybernetics (cat. No.98CH36218) (Vol. 3, pp. 2646-2651). San Diego, CA, USA: IEEE. http://doi.org/10.1109/ICSMC.1998.725059

Franklin, S., Madl, T., Strain, S., Faghihi, U., Dong, D., Kugele, S., .. Chen, S. (2016). A LIDA cognitive model tutorial. Biologically Inspired Cognitive Architectures, 16, 105-130. http://doi.org/10.1016/j.bica.2016.04.003 
Franklin, S., \& Patterson, F. G. (2006). The LIDA architecture: Adding new modes of learning to an intelligent, autonomous, software agent. Pat, 703, 764-1004.

Franklin, S., Strain, S., Snaider, J., McCall, R., \& Faghihi, U. (2012). Global workspace theory, its LIDA model and the underlying neuroscience. Biologically Inspired Cognitive Architectures, 1, 32-43. http://doi.org/10.1016/j.bica. 2012.04 .001

Freeman, W. J. (2003). The wave packet: An action potential for the 21st century. Journal of Integrative Neuroscience, 2(1), 3-30.

Gamez, D. (2008). Progress in machine consciousness. Consciousness and Cognition, 17(3), 887-910. http://doi.org/10.1016/j.concog.2007.04.005

Giessing, C., \& Thiel, C. M. (2012). Pro-cognitive drug effects modulate functional brain network organization. Frontiers in Behavioral Neuroscience, 6. http: //doi.org/10.3389/fnbeh.2012.00053

Glazebrook, J. F., \& Wallace, R. (2009). Small worlds and red queens in the global workspace: An information-theoretic approach. Cognitive Systems Research, 10(4), 333-365. http://doi.org/10.1016/j.cogsys.2009.01.002

González, R. (2007). The chinese room revisited: Artificial intelligence and the nature of mind (PhD thesis). K.U.Leuven. Hoger instituut voor Wijsbegeerte, Leuven. Retrieved from https://lirias.kuleuven.be/handle/1979/939

Graham, G. (2019). Behaviorism. In E. N. Zalta (Ed.), The stanford encyclopedia of philosophy (Spring 2019). Metaphysics Research Lab, Stanford University. Retrieved from https://plato.stanford.edu/archives/spr2019/entries/ behaviorism/

Hagmann, P., Cammoun, L., Gigandet, X., Meuli, R., Honey, C. J., Wedeen, V. J., \& Sporns, O. (2008). Mapping the structural core of human cerebral cortex. PLOS Biology, 6(7), e159. http://doi.org/10.1371/journal.pbio.0060159

Haikonen, P. O. (2003). The cognitive approach to conscious machines. Exeter ; Charlottesville, VA: Imprint Academic.

Haikonen, P. O. (2011). Open questions on shanahan's workspace. International Journal of Machine Consciousness, 03(2), 339-341. http://doi.org/10.1142/ S179384301100073X

Hatfield, G. (2018). René descartes. In E. N. Zalta (Ed.), The stanford encyclopedia of philosophy (Summer 2018). Metaphysics Research Lab, Stanford University. Retrieved from https://plato.stanford.edu/archives/sum2018/entries/ descartes/

Illari, P. M., \& Williamson, J. (2012). What is a mechanism? Thinking about mechanisms across the sciences. European Journal for Philosophy of Science, 2(1), 
119-135. http://doi.org/10.1007/s13194-011-0038-2

Jackson, F. (1982). Epiphenomenal qualia. The Philosophical Quarterly (1950-), 32(127), 127-136. http://doi.org/10.2307/2960077

Jacob, P. (2019). Intentionality. In E. N. Zalta (Ed.), The stanford encyclopedia of philosophy (Spring 2019). Metaphysics Research Lab, Stanford University. Retrieved from https://plato.stanford.edu/archives/spr2019/entries/ intentionality/

Jarman, N., Steur, E., Trengove, C., Tyukin, I. Y., \& van Leeuwen, C. (2017). Self-organisation of small-world networks by adaptive rewiring in response to graph diffusion. Scientific Reports, 7(1), 13158. http://doi.org/10.1038/ s41598-017-12589-9

Kersten, L. (2016). Leveling the field: Talking levels in cognitive science (Text). Carleton University. https://doi.org/10.22215/etd/2016-11382

Koch, C., Massimini, M., Boly, M., \& Tononi, G. (2016). Neural correlates of consciousness: Progress and problems. Nature Reviews Neuroscience, 17(5), 307-321. http://doi.org/10.1038/nrn.2016.22

Lee, U., Oh, G., Kim, S., Noh, G., Choi, B., \& Mashour, G. A. (2010). Brain networks maintain a scale-free organization across consciousness, anesthesia, and recovery: Evidence for adaptive reconfiguration. Anesthesiology, 113(5), 10811091. http://doi.org/10.1097/ALN.0b013e3181f229b5

Lerner, I., Bentin, S., \& Shriki, O. (2012). Spreading activation in an attractor network with latching dynamics: Automatic semantic priming revisited. Cognitive Science, 36(8), 1339-1382. http://doi.org/10.1111/cogs.12007

Machamer, P., Darden, L., \& Craver, C. F. (2000). Thinking about mechanisms. Philosophy of Science, 67(1), 1-25. http://doi.org/10.1086/392759

Mandik, P. (2017). The neurophilosophy of consciousness. In The blackwell companion to consciousness (pp. 458-471). John Wiley \& Sons, Ltd. http: //doi.org/10.1002/9781119132363.ch33

Marr, D. (1982). Vision: A computational investigation into the human representation and processing of visual information. New York: Henry Holt; Company.

McClamrock, R. (1991). Marr's three levels: A re-evaluation. Minds and Machines, 1(2), 185-196. http://doi.org/10.1007/BF00361036

McGinn, C. (1995). Consciousness evaded: Comments on dennett. Philosophical Perspectives, 9, 241-249. http://doi.org/10.2307/2214220

McLaughlin, B., \& Bennett, K. (2018). Supervenience. In E. N. Zalta (Ed.), The stanford encyclopedia of philosophy (Winter 2018). Metaphysics Research Lab, Stanford University. Retrieved from https://plato.stanford.edu/archives/ 
win2018/entries/supervenience/

Metzinger, T. (2000). Neural correlates of consciousness: Empirical and conceptual questions. MIT Press.

Nida-Rümelin, M. (2015). Qualia: The knowledge argument. In E. N. Zalta (Ed.), The stanford encyclopedia of philosophy (Summer 2015). Metaphysics Research Lab, Stanford University. Retrieved from https://plato.stanford. edu/archives/sum2015/entries/qualia-knowledge/

O'Connor, T., \& Wong, H. Y. (2015). Emergent properties. In E. N. Zalta (Ed.), The stanford encyclopedia of philosophy (Summer 2015). Metaphysics Research Lab, Stanford University. Retrieved from https://plato.stanford.edu/archives/ sum2015/entries/properties-emergent/

Oppenheim, P., \& Putnam, H. (1958). Unity of science as a working hypothesis. In R. Boyd, P. Gasper, \& J. D. Trout (Eds.), The philosophy of science.

Oppy, G., \& Dowe, D. (2019). The turing test. In E. N. Zalta (Ed.), The stanford encyclopedia of philosophy (Spring 2019). Metaphysics Research Lab, Stanford University. Retrieved from https://plato.stanford.edu/archives/spr2019/ entriesuring-test/

Oxford English Dictionary. (n.d.). "Desideratum, n.". Retrieved from https: //www. oed.com/view/Entry/50832

Pedersen, T. R. R. (2016). The significance of the body - artificial intellgeince and embodied cognition. Leuven: KU Leuven. Hoger Instituut voor Wijsbegeerte.

Peebles, D., \& Cooper, R. P. (2015). Thirty years after marr's vision: Levels of analysis in cognitive science. Topics in Cognitive Science, 7(2), 187-190. http://doi.org/10.1111/tops.12137

Pitt, D. (2018). Mental representation. In E. N. Zalta (Ed.), The stanford encyclopedia of philosophy (Winter 2018). Metaphysics Research Lab, Stanford University. Retrieved from https://plato.stanford.edu/archives/win2018/ entries/mental-representation/

Poggiolesi, F. (2015). Are the validities of modal logic analytic? Or analyticity again, through information, proof, modal logic and hintikka. Philosophia Scientae, (19), 221-243. http://doi.org/10.4000/philosophiascientiae.1110

Rees, G., \& Frith, C. D. (2017). Methodologies for identifying the neural correlates of consciousness. In S. Schneider \& M. Velmans (Eds.), The blackwell companion to consciousness (pp. 591-606). Chichester, UK: John Wiley \& Sons, Ltd. http://doi.org/10.1002/9781119132363.ch42

Reggia, J. A. (2013). The rise of machine consciousness: Studying consciousness with computational models. Neural Networks, 44, 112-131. http://doi.org/10. 
$1016 / j$.neunet.2013.03.011

Rescorla, M. (2017). The computational theory of mind. In E. N. Zalta (Ed.), The stanford encyclopedia of philosophy (Spring 2017). Metaphysics Research Lab, Stanford University. Retrieved from https://plato.stanford.edu/archives/ spr2017/entries/computational-mind/

Robinson, H. (2017). Dualism. In E. N. Zalta (Ed.), The stanford encyclopedia of philosophy (Fall 2017). Metaphysics Research Lab, Stanford University. Retrieved from https://plato.stanford.edu/archives/fall2017/entries/dualism/

Rosenthal, D. M. (1996). A theory of consciousness. In N. Block, O. Flanagan, \& G. Güzeldere (Eds.), The nature of consciousness (pp. 729-753). Cambridge, MA: MIT Press.

Rosenthal, D. M. (2005). Consciousness and mind. Clarendon Press.

Seth, A. (2007). Models of consciousness. Scholarpedia, 2(1), 1328. http://doi. org/10.4249/scholarpedia.1328

Shanahan, M. (2006). A cognitive architecture that combines internal simulation with a global workspace. Consciousness and Cognition, 15(2), 433-449. http: //doi.org/10.1016/j.concog.2005.11.005

Shanahan, M. (2010). Embodiment and the inner life: Cognition and consciousness in the space of possible minds. Oxford University Press. Retrieved from https: //www . oxfordscholarship.com/view/10.1093/acprof : oso/9780199226559. $001.0001 /$ acprof-9780199226559

Shanahan, M. (2016). The frame problem. In E. N. Zalta (Ed.), The stanford encyclopedia of philosophy (Spring 2016). Metaphysics Research Lab, Stanford University. Retrieved from https://plato.stanford.edu/archives/spr2016/ entries/frame-problem/

Shanahan, M., \& Baars, B. J. (2005). Applying global workspace theory to the frame problem. Cognition, 98(2), 157-176. http://doi.org/10.1016/j.cognition. 2004.11 .007

Sporns, O. (2010). Networks of the brain. MIT Press.

Sporns, O., \& Zwi, J. D. (2004). The small world of the cerebral cortex. Neuroinformatics, 2(2), 145-162. http://doi.org/10.1385/NI:2:2:145

Standing, L. (1973). Learning 10000 pictures. Quarterly Journal of Experimental Psychology, 25(2), 207-222. http://doi.org/10.1080/14640747308400340

Swinney, D. A. (1979). Lexical access during sentence comprehension: (Re)consideration of context effects. Journal of Verbal Learning and Verbal Behavior, 18(6), 645-659. http://doi.org/10.1016/S0022-5371(79)90355-4 
Tahko, T. E., \& Lowe, E. J. (2016). Ontological dependence. In E. N. Zalta (Ed.), The stanford encyclopedia of philosophy (Winter 2016). Metaphysics Research Lab, Stanford University. Retrieved from https://plato.stanford.edu/archives/ win2016/entries/dependence-ontological/

Turing, A. M. (1950). Computing machinery and intelligence. Mind, LIX(236), 433-460. http://doi.org/10.1093/mind/LIX.236.433

Tye, M. (2018). Qualia. In E. N. Zalta (Ed.), The stanford encyclopedia of philosophy (Summer 2018). Metaphysics Research Lab, Stanford University. Retrieved from https://plato.stanford.edu/archives/sum2018/entries/qualia/

van Benthem, J., \& van Rooy, R. (2003). Connecting the different faces of information. Journal of Logic, Language and Information, 12(4), 375-379. http://doi.org/ 10.1023/A: 1025026116766

Van Gelder, T. (1995). What might cognition be, if not computation? The Journal of Philosophy, 92(7), 345-381. http://doi.org/10.2307/2941061

Van Gulick, R. (2018). Consciousness. In E. N. Zalta (Ed.), The stanford encyclopedia of philosophy (Spring 2018). Metaphysics Research Lab, Stanford University. Retrieved from https://plato.stanford.edu/archives/spr2018/entries/ consciousness/

Varzi, A. (2019). Mereology. In E. N. Zalta (Ed.), The stanford encyclopedia of philosophy (Spring 2019). Metaphysics Research Lab, Stanford University. Retrieved from https://plato.stanford.edu/archives/spr2019/entries/ mereology/

Wallace, R. (2005). Consciousness: A mathematical treatment of the global neuronal workspace model. New York: Springer.

Watts, D. J., \& Strogatz, S. H. (1998). Collective dynamics of "small-world" networks. Nature, 393(6684), 440-442. http://doi.org/10.1038/30918

Wilberforce Clarke, H. (1891). Divan-e Hafez. Calcutta, India. 


\title{
Master's thesis filing card
}

\author{
Student: MohammadHossein Manuel Haqiqatkhah
}

Title: Machine Consciousness and the Global Workspace Theory

Dutch title: Het Bewustzijn van Machines en de Global Workspace Theorie

$U D C: 681.3^{*} \mathrm{I} 20$

\section{Abstract:}

The past century has seen a resurgence of interest in the study of consciousness among scholars of various fields, from philosophy to psychology and neuroscience. Since the birth of Artificial Intelligence in the 1950s, the study of consciousness in machines has received an increasing amount of attention in computer science that gave rise to the new field of machine consciousness (MC). Meanwhile, interdisciplinary research in philosophy, neuroscience, and cognitive science has advanced neurocognitive theories for consciousness. Among many models proposed for consciousness, the Global Workspace Theory (GWT) is a promising theory of consciousness that has received a staggering amount of philosophical and empirical support in the past decades. This dissertation discusses the GWT and its potentials for MC from a mechanistic point of view. To do so, Chapter 1 gives an overview of the philosophical study of consciousness and the history of MC. Then, in Chapter 2, mechanistic explanations and tri-level models are introduced, which provide a robust framework to construct and assay various theories of consciousness. In Chapter 3, neural correlates (and thereby, neurocognitive theories) of consciousness are introduced. This chapter presents the GWT in details and, along with its strengths, discusses the philosophical issues it raises. Chapter 4 addresses two computational implementations of the GWT (viz., IDA and LIDA) which satisfy specific goals of MC. Finally, in Chapter 5, one of the philosophical problems of MC, namely, the Frame Problem (FP), is introduced. It is argued that the architectures based on the GWT are immune to the FP. The chapter concludes that the GWT is capable of "solving" the FP, and discusses its implications for MC and the computational theory of mind. Chapter 6 wraps up the dissertation by reviewing the content.

Thesis submitted for the degree of Master of Science in Artificial Intelligence, option Engineering and Computer Science

Thesis supervisor: Prof. Roger Vergauwen

Assessors: Prof. Bettina Berendt

Prof. Danny De Schreye

Mentor: 